\title{
Structured Factorizations in Scalar Product
} Spaces

Mackey, D.S. and Mackey, N. and Tisseur, F. 2006

MIMS EPrint: 2007.220

Manchester Institute for Mathematical Sciences

School of Mathematics

The University of Manchester

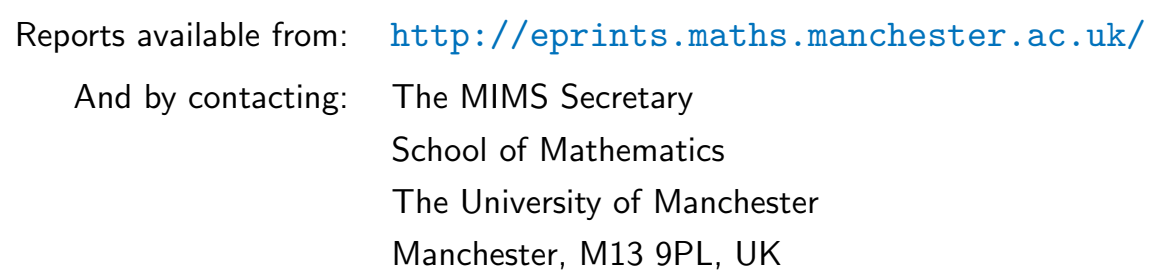

ISSN 1749-9097 


\title{
STRUCTURED FACTORIZATIONS IN SCALAR PRODUCT SPACES*
}

\author{
D. STEVEN MACKEY ${ }^{\dagger}$, NILOUFER MACKEY ${ }^{\ddagger}$, AND FRANÇOISE TISSEUR ${ }^{\dagger}$
}

\begin{abstract}
Let $A$ belong to an automorphism group, Lie algebra, or Jordan algebra of a scalar product. When $A$ is factored, to what extent do the factors inherit structure from $A$ ? We answer this question for the principal matrix square root, the matrix sign decomposition, and the polar decomposition. For general $A$, we give a simple derivation and characterization of a particular generalized polar decomposition, and we relate it to other such decompositions in the literature. Finally, we study eigendecompositions and structured singular value decompositions, considering in particular the structure in eigenvalues, eigenvectors, and singular values that persists across a wide range of scalar products.

A key feature of our analysis is the identification of two particular classes of scalar products, termed unitary and orthosymmetric, which serve to unify assumptions for the existence of structured factorizations. A variety of different characterizations of these scalar product classes are given.

Key words. automorphism group, Lie group, Lie algebra, Jordan algebra, bilinear form, sesquilinear form, scalar product, indefinite inner product, orthosymmetric, adjoint, factorization, symplectic, Hamiltonian, pseudo-orthogonal, polar decomposition, matrix sign function, matrix square root, generalized polar decomposition, eigenvalues, eigenvectors, singular values, structure preservation
\end{abstract}

AMS subject classifications. 15A18, 15A21, 15A23, 15A57, 15A63

DOI. $10.1137 / 040619363$

1. Introduction. The factorization of a general matrix into a product of structured factors plays a key role in theoretical and computational linear algebra. In this work we address the following question: if we apply one of the standard factorizations to a matrix that is already structured, to what extent do the factors have additional structure related to that of the original matrix?

Many applications generate structure, and there are potential benefits to be gained by exploiting it when developing theory and deriving algorithms. For example, algorithms that preserve structure may have reduced storage requirements and operation counts, may be more accurate, and may also provide solutions that are physically more meaningful in the presence of rounding and truncation errors.

The structured matrices we consider belong to the automorphism group $\mathbb{G}$, the Lie algebra $\mathbb{L}$, and the Jordan algebra $\mathbb{J}$ associated with a scalar product, that is, a nondegenerate bilinear or sesquilinear form on $\mathbb{K}^{n}$. These classes of matrices include linear structures such as complex symmetric, pseudosymmetric, and Hamiltonian matrices, as well as nonlinear structures such as complex orthogonal, pseudo-orthogonal and symplectic matrices. Section 2 introduces concepts and notation needed for our unified treatment of structured factorizations in scalar product spaces. We introduce two important special types of scalar products, termed unitary and orthosymmetric,

*Received by the editors November 22, 2004; accepted for publication (in revised form) by P. Benner June 30, 2005; published electronically January 27, 2006.

http://www.siam.org/journals/simax/27-3/61936.html

†School of Mathematics, The University of Manchester, Sackville Street, Manchester, M60 1QD, UK (smackey@ma.man.ac.uk, ftisseur@ma.man.ac.uk, http://www.ma.man.ac.uk/ ftisseur/). The work of the first and third authors was supported by Engineering and Physical Sciences Research Council grant GR/S31693. The work of the third author was also supported by grant GR/R45079.

¥Department of Mathematics, Western Michigan University, Kalamazoo, MI 49008 (nil.mackey@ wmich.edu, http://homepages.wmich.edu/ ${ }^{\sim}$ mackey/). The work of this author was supported by Engineering and Physical Sciences Research Council Visiting Fellowship GR/S15563. 
and describe several equivalent ways to characterize them. The proofs of these equivalences have been delegated to an appendix to avoid disrupting the flow of the paper. These characterizations are essential in clarifying existing results in the literature and in formulating simple, unified assumptions on the scalar product that guarantee the existence of structured factors.

The factorizations we study are the principal square root, the matrix sign decomposition, the polar decomposition, and the generalized polar decomposition (i.e., polar decomposition with respect to a general scalar product). Our results in sections 3 and 4 show that structured principal square roots and structured factors for the matrix sign decomposition exist in arbitrary scalar product spaces. As shown in sections 5 and 6 , structured polar factors are guaranteed to exist whenever the scalar product is unitary, while the generalized polar decomposition always exists in an orthosymmetric scalar product space.

Much work has been done on structured spectral decompositions, Schur-like forms, and Jordan-like canonical forms for matrices arising in the context of a specific scalar product or restricted classes of scalar products. An overall unified structured eigendecomposition theory for all scalar products appears to be difficult. However, we show in section 7 that even in an arbitrary scalar product space, the eigenvalues and eigenvectors of matrices in $\mathbb{G}, \mathbb{L}$, and $\mathbb{J}$ have a significant level of structure.

Finally, we discuss in section 8 the extent to which the singular values of matrices in $\mathbb{G}, \mathbb{L}$, or $\mathbb{J}$ are structured and survey what is known about structured singular value decompositions (SVDs).

2. Scalar products and structured matrices. We begin with the basic definitions and properties of scalar products, and the structured classes of matrices associated with them.

2.1. Scalar products. Consider scalar-valued maps from $\mathbb{K}^{n} \times \mathbb{K}^{n}$ to $\mathbb{K}:(x, y) \mapsto$ $\langle x, y\rangle$, where $\mathbb{K}$ denotes the field $\mathbb{R}$ or $\mathbb{C}$. When such maps are linear in each argument they are called bilinear forms on $\mathbb{K}^{n}$. For $\mathbb{K}=\mathbb{C}$, maps that are conjugate linear in the first argument and linear in the second are called sesquilinear forms.

A real or complex bilinear form has a unique matrix representation given by $\langle x, y\rangle=x^{T} M y$, while a sesquilinear form can be represented by $\langle x, y\rangle=x^{*} M y$. We will denote $\langle x, y\rangle$ by $\langle x, y\rangle_{\mathrm{M}}$ as needed. Note that $\langle\cdot, \cdot\rangle_{\mathrm{M}}$ is nondegenerate if and only if $M$ is nonsingular. For brevity, the term scalar product will be used to refer to any nondegenerate bilinear or sesquilinear form on $\mathbb{K}^{n}$. The space $\mathbb{K}^{n}$ equipped with a fixed scalar product is said to be a scalar product space.

2.2. Adjoints, automorphisms, and algebras. To each scalar product there corresponds a notion of adjoint, generalizing the idea of transpose ${ }^{T}$ and conjugate transpose ${ }^{*}$. That is, for any operator $A$ there exists a unique operator $A^{\star}$, called the adjoint of $A$ with respect to the scalar product $\langle\cdot, \cdot\rangle_{\mathrm{M}}$, such that

$$
\langle A x, y\rangle_{\mathrm{M}}=\left\langle x, A^{\star} y\right\rangle_{\mathrm{M}} \quad \text { for all } x, y \in \mathbb{K}^{n} .
$$

An explicit formula for the adjoint is given by

$$
A^{\star}= \begin{cases}M^{-1} A^{T} M & \text { for bilinear forms, } \\ M^{-1} A^{*} M & \text { for sesquilinear forms. }\end{cases}
$$

This immediately yields the following useful result. Here $A \sim B$ denotes similarity between matrices $A$ and $B$. 
Lemma 2.1. For real or complex bilinear forms, $A^{\star} \sim A$, while for sesquilinear forms, $A^{\star} \sim \bar{A}$.

As one might expect, the adjoint has a number of properties analogous to those of transpose. The following basic properties hold for all scalar products. We omit the simple proofs.

$$
\begin{aligned}
& (A+B)^{\star}=A^{\star}+B^{\star},(A B)^{\star}=B^{\star} A^{\star},\left(A^{-1}\right)^{\star}=\left(A^{\star}\right)^{-1} \\
& (\alpha A)^{\star}=\alpha A^{\star} \text { for bilinear forms, }(\alpha A)^{\star}=\bar{\alpha} A^{\star} \text { for sesquilinear forms. }
\end{aligned}
$$

Notice the absence of the involutory property $\left(A^{\star}\right)^{\star}=A$ from this list. Only a restricted class of scalar products have an involutory adjoint, as discussed in Appendix A.

Associated to each scalar product are three classes of structured matrices: the automorphisms, self-adjoint matrices, and skew-adjoint matrices. A matrix $G$ is said to be an automorphism (or isometry) of the scalar product $\langle\cdot, \cdot\rangle_{\mathrm{M}}$ if $\langle G x, G y\rangle_{\mathrm{M}}=$ $\langle x, y\rangle_{\mathrm{M}}$ for all $x, y \in \mathbb{K}^{n}$, or equivalently, $\langle G x, y\rangle_{\mathrm{M}}=\left\langle x, G^{-1} y\right\rangle_{\mathrm{M}}$ for all $x, y \in \mathbb{K}^{n}$. In terms of adjoint this corresponds to $G^{\star}=G^{-1}$. Thus, the automorphism group of $\langle\cdot, \cdot\rangle_{\mathrm{M}}$ is the set

$$
\mathbb{G}=\left\{G \in \mathbb{K}^{n \times n}: G^{\star}=G^{-1}\right\} .
$$

Matrices $S$ that are self-adjoint with respect to the scalar product, i.e., $\langle S x, y\rangle_{\mathrm{M}}=$ $\langle x, S y\rangle_{\mathrm{M}}$ for all $x, y \in \mathbb{K}^{n}$, form a Jordan algebra,

$$
\mathbb{J}=\left\{S \in \mathbb{K}^{n \times n}: S^{\star}=S\right\}
$$

and matrices $K$ that are skew-adjoint with respect to the scalar product, i.e., $\langle K x, y\rangle_{\mathrm{M}}=$ $-\langle x, K y\rangle_{\mathrm{M}}$ for all $x, y \in \mathbb{K}^{n}$, belong to the Lie algebra $\mathbb{L}$, defined by

$$
\mathbb{L}=\left\{K \in \mathbb{K}^{n \times n}: K^{\star}=-K\right\} .
$$

Some important instances of $\mathbb{G}, \mathbb{L}$ and $\mathbb{J}$ are listed in Table 2.1 .

$\mathbb{G}$ always forms a multiplicative group (indeed a Lie group), although it is not a linear subspace. By contrast, the sets $\mathbb{L}$ and $\mathbb{J}$ are linear subspaces, but they are not closed under multiplication. Instead $\mathbb{L}$ is closed with respect to the Lie bracket $\left[K_{1}, K_{2}\right]=K_{1} K_{2}-K_{2} K_{1}$, while $\mathbb{J}$ is closed with respect to the Jordan product $\left\{S_{1}, S_{2}\right\}=\frac{1}{2}\left(S_{1} S_{2}+S_{2} S_{1}\right)$. Technical correctness dictates that the symbols $\star, \mathbb{G}, \mathbb{L}$, and $\mathbb{J}$ all bear the subscript ${ }_{\mathrm{M}}$. For the sake of readability, however, we will usually suppress this subscript.

$\mathbb{G}$ is always closed under products and inverses, being a multiplicative group. The following closure properties of $\mathbb{L}$ and $\mathbb{I}$ will be useful; we omit the simple proofs.

LEMMA 2.2.

(a) Inverses: For nonsingular $A, A \in \mathbb{L} \Rightarrow A^{-1} \in \mathbb{L}$ and $A \in \mathbb{J} \Rightarrow A^{-1} \in \mathbb{J}$.

(b) Powers: $A \in \mathbb{J} \Rightarrow A^{n} \in \mathbb{J}$ for all $n \in \mathbb{N} . A \in \mathbb{L} \Rightarrow A^{n} \in\left\{\begin{array}{l}\mathbb{L} \text { for } n \text { odd, } \\ \mathbb{J} \text { for } n \text { even. }\end{array}\right.$

(c) Polynomials: For bilinear forms, $\mathbb{L}$ is closed under all odd polynomials over $\mathbb{K}$, while $\mathbb{J}$ is closed under all polynomials over $\mathbb{K}$. When the scalar product is sesquilinear, the same results hold only for real polynomials.

(d) Linear combinations: For bilinear forms, $\mathbb{L}$ and $\mathbb{J}$ are closed under arbitrary $\mathbb{K}$-linear combinations and hence are $\mathbb{K}$-linear subspaces. However, for sesquilinear forms $\mathbb{L}$ and $\mathbb{J}$ are closed only under real linear combinations and hence are only $\mathbb{R}$-linear subspaces. 
(e) Commuting products: Suppose $A$ and $B$ commute. Then

$$
\begin{aligned}
A, B \in \mathbb{L} & \Longrightarrow A B \in \mathbb{J}, \\
A, B \in \mathbb{J} & \Longrightarrow A B \in \mathbb{J}, \\
A \in \mathbb{L}, B \in \mathbb{J} & \Longrightarrow A B \in \mathbb{L}, B A \in \mathbb{L} .
\end{aligned}
$$

In general, noncommuting products retain neither $\mathbb{L}$ nor $\mathbb{J}$ structure.

2.3. Unitary and orthosymmetric scalar products. We aim to present results on structured factorizations that use a minimum number of hypotheses on the scalar product defining the structures. As shown in the next sections, some results on structured factorizations need no assumptions on the scalar product at all, while others require at least one of the following properties:

(a) $\left(A^{*}\right)^{\star}=\left(A^{\star}\right)^{*}$ for all $A \in \mathbb{K}^{n \times n}$.

(b) Adjoint preserves unitarity; that is, $U$ unitary $\Rightarrow U^{\star}$ is unitary.

(c) Adjoint preserves Hermitian positive (semi)definite structure; that is, $H$ Hermitian positive (semi)definite $\Rightarrow H^{\star}$ is Hermitian positive (semi)definite.

(d) $M=\beta U$ for some unitary $U$ and $\beta>0$.

(e) Adjoint with respect to $\langle\cdot, \cdot\rangle_{\mathrm{M}}$ is involutory; that is, $\left(A^{\star}\right)^{\star}=A$ for all $A \in$ $\mathbb{K}^{n \times n}$.

(e') $\left(A^{\star}\right)^{\star}=A$ for all $A$ in some CS-set ${ }^{1}$ for $\mathbb{K}^{n \times n}$.

(f) $\mathbb{K}^{n \times n}=\mathbb{L} \oplus \mathbb{J}$.

(g) For bilinear forms, $M^{T}= \pm M$. For sesquilinear forms, $M^{*}=\alpha M$ with $\alpha \in \mathbb{C},|\alpha|=1$.

(h) Vector orthogonality is a symmetric relation; that is,

$$
\langle x, y\rangle_{\mathrm{M}}=0 \Longleftrightarrow\langle y, x\rangle_{\mathrm{M}}=0 \quad \text { for all } x, y \in \mathbb{K}^{n} \text {. }
$$

At first glance there is no obvious connection or relationship among these conditions. However, it turns out that they cluster together into two groups of equivalent properties,

$$
(\mathrm{a}) \Leftrightarrow(\mathrm{b}) \Leftrightarrow(\mathrm{c}) \Leftrightarrow(\mathrm{d}) \quad \text { and } \quad(\mathrm{e}) \Leftrightarrow\left(\mathrm{e}^{\prime}\right) \Leftrightarrow(\mathrm{f}) \Leftrightarrow(\mathrm{g}) \Leftrightarrow(\mathrm{h})
$$

These equivalences are proved in Appendix A, together with the equivalence of some additional properties. We can now identify two natural classes of scalar products.

Definition 2.3. A scalar product is said to be unitary if it satisfies any one (and hence all) of the properties (a), (b), (c), (d) above.

Definition 2.4. A scalar product is said to be orthosymmetric if it satisfies any one (and hence all) of the properties (e), (e'), (f), (g), (h) above.

The name "orthosymmetric" has been adopted in Definition 2.4 because of property (h). Note that all the "classical" examples of scalar products listed in Table 2.1 are both orthosymmetric and unitary.

We like to abbreviate property (a) by saying, "In unitary scalar products, the stars commute." This commutativity leads to another useful closure property needed for the structured polar decomposition.

Proposition 2.5. For any unitary scalar product with automorphism group $\mathbb{G}$, Lie algebra $\mathbb{L}$, and Jordan algebra $\mathbb{J}$, we have $A \in \mathbb{S} \Rightarrow A^{*} \in \mathbb{S}$, where $\mathbb{S}=\mathbb{G}, \mathbb{L}$ or $\mathbb{J}$.

Proof. We consider only the case when $\mathbb{S}=\mathbb{G}$, as the proof for $\mathbb{S}=\mathbb{L}$ or $\mathbb{S}=\mathbb{J}$ is similar. Since the stars commute in a unitary scalar product space, $A \in \mathbb{G} \Rightarrow A^{\star}=$ $A^{-1} \Rightarrow\left(A^{\star}\right)^{*}=\left(A^{-1}\right)^{*} \Rightarrow\left(A^{*}\right)^{\star}=\left(A^{*}\right)^{-1} \Rightarrow A^{*} \in \mathbb{G}$.

\footnotetext{
${ }^{1} \mathrm{~A} C S$-set for $\mathbb{K}^{n \times n}$ is any set of matrices $\mathcal{S} \subseteq \mathbb{K}^{n \times n}$ such that $B S=S B$ for all $S \in \mathcal{S}$ implies $B=\alpha I$ for some $\alpha \in \mathbb{K}$. For example, any open subset $\mathcal{S} \subseteq \mathbb{K}^{n \times n}$ is a CS-set (see Lemma A.2(e)).
} 
TABLE 2.1

A sampling of structured matrices associated with scalar products $\langle\cdot, \cdot\rangle_{\mathrm{M}}$, where $\mathrm{M}$ is the matrix defining the scalar product.

\begin{tabular}{|c|c|c|c|c|}
\hline Space & M & $\begin{array}{l}\text { Automorphism group } \\
\mathbb{G}=\left\{G: G^{\star}=G^{-1}\right\}\end{array}$ & $\begin{array}{c}\text { Jordan algebra } \\
\mathbb{J}=\left\{S: S^{\star}=S\right\}\end{array}$ & $\begin{aligned} & \text { Lie algebra } \\
& \mathbb{L}=\left\{K: K^{\star}=-K\right\}\end{aligned}$ \\
\hline \multicolumn{5}{|c|}{ Bilinear forms } \\
\hline $\mathbb{R}^{n}$ & $I$ & real orthogonals & symmetrics & skew-symmetrics \\
\hline $\mathbb{C}^{n}$ & $I$ & complex orthogonals & complex symmetrics & cplx. skew-symmetrics \\
\hline $\mathbb{R}^{n}$ & $\Sigma_{p, q}$ & pseudo-orthogonals $^{\mathrm{a}}$ & pseudosymmetrics & pseudoskew-symmetrics \\
\hline $\mathbb{C}^{n}$ & $\Sigma_{p, q}$ & cplx. pseudo-orthogonals & cplx. pseudosymm. & cplx. pseudoskew-symm. \\
\hline $\mathbb{R}^{n}$ & $R$ & real perplectics & persymmetrics & perskew-symmetrics \\
\hline $\mathbb{R}^{2 n}$ & $J$ & real symplectics & skew-Hamiltonians & Hamiltonians \\
\hline $\mathbb{C}^{2 n}$ & $J$ & complex symplectics & cplx. $J$-skew-symm. & complex $J$-symmetrics \\
\hline
\end{tabular}

\begin{tabular}{|c|c|c|c|c|}
\hline \multicolumn{5}{|c|}{ Sesquilinear forms } \\
\hline $\mathbb{C}^{n}$ & $I$ & unitaries & Hermitian & skew-Hermitian \\
\hline $\mathbb{C}^{n}$ & $\Sigma_{p, q}$ & pseudo-unitaries $^{\mathrm{b}}$ & pseudo-Hermitian & pseudoskew-Hermitian \\
\hline $\mathbb{C}^{2 n}$ & $J$ & conjugate symplectics & $J$-skew-Hermitian & $J$-Hermitian \\
\hline
\end{tabular}

Here, $R=\left[{ }_{1} \cdot{ }^{1}\right], \quad J=\left[\begin{array}{cc}0 & I_{n} \\ -I_{n} & 0\end{array}\right], \quad \Sigma_{p, q}=\left[\begin{array}{cc}I_{p} & 0 \\ 0 & -I_{q}\end{array}\right] \in \mathbb{R}^{n \times n}$.

\footnotetext{
a Physicists refer to the pseudo-orthogonal group with $\Sigma_{3,1}=\left[\begin{array}{ll}I_{3} & \\ & -1\end{array}\right]$ as the Lorentz group.

${ }^{\mathrm{b}}$ Pseudo-unitary matrices are sometimes called $\Sigma_{p, q}$-unitaries, or hypernormal matrices in the signal processing literature.
}

This result allows us to say that in a unitary scalar product space, $\mathbb{G}, \mathbb{L}$, and $\mathbb{J}$ are all "closed under the stars."

3. Structured square roots. A matrix $X \in \mathbb{K}^{n \times n}$ such that $A=X^{2}$ is said to be a square root of $A$. Much is known about the existence of matrix square roots and their computation; see [4], [21], [26], for example. In general, the number of square roots may be zero, finite, or infinite. When a square root does exist, the one of most significance for theory and computation is the principal square root, identified by having all its eigenvalues in the open right half-plane and denoted by $A^{1 / 2}$. It is well known that $A^{1 / 2}$ exists if and only if $A$ has no eigenvalues on $\mathbb{R}^{-}:=\{x \in \mathbb{R}: x \leq 0\}$, and that $A^{1 / 2}$ is unique whenever it exists.

If a structured matrix $A$ has a principal square root with the same or related structure, then we can view the equation $A=A^{1 / 2} \cdot A^{1 / 2}$ as a structured factorization. Square roots with structure related to the original matrix have been studied in, for example, [17], [24]. In this section we address the question of whether the principal square root of a matrix in $\mathbb{G}, \mathbb{L}$, or $\mathbb{J}$ associated with an arbitrary scalar product is also structured. The answer will have a significant impact on many of the other structured factorizations considered in this paper.

Lemma 3.1. Suppose $A$ has a principal square root, and let $A^{\star}$ denote the adjoint of $A$ with respect to an arbitrary scalar product (bilinear or sesquilinear). Then

(a) $\left(A^{\star}\right)^{1 / 2}$ and $\left(A^{-1}\right)^{1 / 2}$ both exist,

(b) $\left(A^{\star}\right)^{1 / 2}=\left(A^{1 / 2}\right)^{\star}$ and $\left(A^{-1}\right)^{1 / 2}=\left(A^{1 / 2}\right)^{-1}$. 
Proof. (a) $A$ has no eigenvalues on $\mathbb{R}^{-}$, so neither does $A^{\star}$, since by Lemma 2.1 $A^{\star}$ is similar to either $A$ or $\bar{A}$. Now $A^{-1}$ also has no eigenvalues on $\mathbb{R}^{-}$, since the eigenvalues of $A^{-1}$ are the reciprocals of those of $A$. Hence $A^{1 / 2},\left(A^{\star}\right)^{1 / 2}$, and $\left(A^{-1}\right)^{1 / 2}$ all exist.

(b) $\left(A^{1 / 2}\right)^{\star}$ is a square root for $A^{\star}$, since $\left(A^{1 / 2}\right)^{\star} \cdot\left(A^{1 / 2}\right)^{\star}=\left(A^{1 / 2} \cdot A^{1 / 2}\right)^{\star}=A^{\star}$. But $\left(A^{1 / 2}\right)^{\star}$, being similar to either $A^{1 / 2}$ or $\overline{A^{1 / 2}}$, has all its eigenvalues in the open right half-plane and hence must be the principal square root of $A^{\star}$. The proof of the second part of part (b) is similar.

THEOREM 3.2 (structured principal square roots). Suppose $\mathbb{G}, \mathbb{L}$, and $\mathbb{J}$ are the automorphism group, Lie algebra, and Jordan algebra, respectively, of an arbitrary scalar product and $A$ is a matrix that has a principal square root. Then

(a) $A \in \mathbb{G} \Longrightarrow A^{1 / 2} \in \mathbb{G}$,

(b) $A \in \mathbb{L} \Longrightarrow A^{1 / 2}$ is not in $\mathbb{L}$,

(c) $A \in \mathbb{J} \Longrightarrow A^{1 / 2} \in \mathbb{J}$.

Proof. Using Lemma 3.1, we have

(a) $A \in \mathbb{G} \Rightarrow A^{\star}=A^{-1} \Rightarrow\left(A^{\star}\right)^{1 / 2}=\left(A^{-1}\right)^{1 / 2} \Rightarrow\left(A^{1 / 2}\right)^{\star}=\left(A^{1 / 2}\right)^{-1} \Rightarrow$ $A^{1 / 2} \in \mathbb{G}$.

(b) Suppose $A^{1 / 2} \in \mathbb{L}$. Then $A=\left(A^{1 / 2}\right)^{2} \in \mathbb{J}$, since the square of any matrix in $\mathbb{L}$ is in $\mathbb{J}$. But $\mathbb{L} \cap \mathbb{J}=\{0\}$; thus $A=0$, contradicting the existence of $A^{1 / 2}$.

(c) $A \in \mathbb{J} \Rightarrow A^{\star}=A \Rightarrow\left(A^{\star}\right)^{1 / 2}=A^{1 / 2} \Rightarrow\left(A^{1 / 2}\right)^{\star}=A^{1 / 2} \Rightarrow A^{1 / 2} \in \mathbb{J}$.

In section 5.2 we need to consider square roots of structured matrices that are singular and hence do not have a principal square root. These singular matrices, though, are Hermitian positive semidefinite and thus have a distinguished square root - the unique Hermitian positive semidefinite square root [25]. Note that whenever a matrix $A$ has both a principal square root and a Hermitian positive semidefinite square root, these two roots coincide, and so without ambiguity we can use $A^{1 / 2}$ to denote this unique distinguished square root. In the following analogues of Lemma 3.1 and Theorem 3.2, we consider only unitary scalar products.

Lemma 3.3. Suppose $A$ is Hermitian positive semidefinite, and let $A^{\star}$ denote the adjoint of $A$ with respect to a unitary scalar product. Then

(a) $\left(A^{\star}\right)^{1 / 2}$ exists, and $\left(A^{\star}\right)^{1 / 2}=\left(A^{1 / 2}\right)^{\star}$.

(b) If $A$ is nonsingular, then $\left(A^{-1}\right)^{1 / 2}$ exists and $\left(A^{-1}\right)^{1 / 2}=\left(A^{1 / 2}\right)^{-1}$.

Proof. (a) $A$ and $A^{1 / 2}$ are both Hermitian positive semidefinite; hence by Definition $2.3(\mathrm{c})$ so are both $A^{\star}$ and $\left(A^{1 / 2}\right)^{\star}$. Thus $\left(A^{\star}\right)^{1 / 2}$ exists. Since $\left(A^{1 / 2}\right)^{\star} \cdot\left(A^{1 / 2}\right)^{\star}=$ $\left(A^{1 / 2} \cdot A^{1 / 2}\right)^{\star}=A^{\star},\left(A^{1 / 2}\right)^{\star}$ must be the unique Hermitian positive semidefinite square root of $A^{\star}$, i.e., $\left(A^{\star}\right)^{1 / 2}=\left(A^{1 / 2}\right)^{\star}$.

(b) If $A$ is nonsingular, then the Hermitian positive semidefinite square root is the same as the principal square root; thus Lemma 3.1 applies.

Theorem 3.4 (structured Hermitian positive semidefinite square roots). Suppose $\mathbb{G}, \mathbb{L}$, and $\mathbb{J}$ are the automorphism group, Lie algebra, and Jordan algebra, respectively, of a unitary scalar product, and $A$ is Hermitian positive semidefinite. Then

(a) $A \in \mathbb{G} \Longrightarrow A^{1 / 2} \in \mathbb{G}$,

(b) $A \in \mathbb{L} \Longrightarrow A^{1 / 2} \in \mathbb{L}$ only if $A=0$,

(c) $A \in \mathbb{J} \Longrightarrow A^{1 / 2} \in \mathbb{J}$.

Proof. Using Lemma 3.3 in place of Lemma 3.1, the proofs are all formally identical to the corresponding ones in Theorem 3.2. The only difference is that $(\cdot)^{1 / 2}$ denotes the Hermitian positive semidefinite square root.

4. Structured matrix sign decomposition. The matrix sign function, defined for matrices $A \in \mathbb{C}^{n \times n}$ with no pure imaginary eigenvalues, was originally 
introduced and developed by Roberts [47] as a tool to solve algebraic Riccati equations. In [20], Higham extended this notion to a factorization $A=S N$, where $S$ is the matrix sign of $A$ and $N:=S^{-1} A$, and showed how this "matrix sign decomposition" is closely related to the polar decomposition. The next result shows that for a matrix $A$ structured with respect to an arbitrary scalar product, the sign factors $S$ and $N$ are also structured.

THEOREM 4.1. Let $\mathbb{S}$ be the automorphism group $\mathbb{G}$, the Lie algebra $\mathbb{L}$, or the Jordan algebra $\mathbb{J}$ of an arbitrary scalar product, and suppose $A \in \mathbb{K}^{n \times n}$ has a sign decomposition $A=S N$. Then

$$
A \in \mathbb{S} \Longrightarrow S \in \mathbb{S} \text { and } N \in \begin{cases}\mathbb{S} & \text { when } \mathbb{S}=\mathbb{G}, \mathbb{J}, \\ \mathbb{J} & \text { when } \mathbb{S}=\mathbb{L} .\end{cases}
$$

Proof. In each case we focus first on the structure of the factor $N$ and then from this deduce the structure of $S$, using the characterizations $N=\left(A^{2}\right)^{1 / 2}$ and $S=A\left(A^{2}\right)^{-1 / 2}$ from [20]. Note that $A$ has a matrix sign if and only if $A$ has no eigenvalues on the imaginary axis, so that $A^{2}$ then has no zero or negative eigenvalues, and hence $\left(A^{2}\right)^{1 / 2}$ exists.

First suppose that $A \in \mathbb{G}$. Then $A^{2} \in \mathbb{G}$, so $N=\left(A^{2}\right)^{1 / 2} \in \mathbb{G}$ by Theorem 3.2. Since $\mathbb{G}$ is a group, it follows that $S=A N^{-1}$ is also in $\mathbb{G}$.

Next let $A \in \mathbb{L}$. Then $A^{2} \in \mathbb{J}$ by Lemma 2.2(b); hence $N=\left(A^{2}\right)^{1 / 2} \in \mathbb{J}$ by Theorem 3.2, and thus $N^{-1} \in \mathbb{J}$ by Lemma 2.2(a). Finally, since $A$ and $N^{-1}$ commute, we have $S=A N^{-1} \in \mathbb{L}$ by Lemma 2.2(e).

Finally, consider $A \in \mathbb{J}$. Then $A^{2} \in \mathbb{J}$, and thus $N=\left(A^{2}\right)^{1 / 2} \in \mathbb{J}$ and $N^{-1} \in \mathbb{J}$ just as above. With $A$ and $N^{-1}$ being commuting elements of $\mathbb{J}$, we have $S=A N^{-1} \in \mathbb{J}$ by Lemma 2.2(e).

Theorem 4.1 can also be proved using globally convergent structure-preserving iterations for the matrix sign. Such iterations $X_{k+1}=f\left(X_{k}\right)$ converge to $\operatorname{sign}\left(X_{0}\right)$ for any $X_{0} \in \mathbb{S}$ for which $\operatorname{sign}\left(X_{0}\right)$ is defined, and have $X_{k} \in \mathbb{S}$ for all $k$. A family of such iterations is discussed in [23], but only in the context of preserving automorphism group structure. Two particular examples are the cubically converging $X_{k+1}=f\left(X_{k}\right)=X_{k}\left(3 I+X_{k}^{2}\right)\left(I+3 X_{k}^{2}\right)^{-1}$ and the quintically converging $Y_{k+1}=g\left(Y_{k}\right)=Y_{k}\left(5 I+10 Y_{k}^{2}+Y_{k}^{4}\right)\left(I+10 Y_{k}^{2}+5 Y_{k}^{4}\right)^{-1}$. That the matrix functions $f$ and $g$ preserve automorphism group structure is shown in [23] and [24], but by using Lemma 2.2 they can also be shown to preserve $\mathbb{L}$ and $\mathbb{J}$ structure. The well-known Newton iteration $Z_{k+1}=\frac{1}{2}\left(Z_{k}+Z_{k}^{-1}\right)$ for matrix sign also preserves $\mathbb{L}$ and $\mathbb{J}$. The existence of these structure-preserving iterations gives perhaps a more direct insight into why the sign $S$ of a matrix $A$ should have the same structure as $A$. The fact that $A, S$, and $N$ all commute then implies that $N \in \mathbb{J}$ whenever $A \in \mathbb{L}$ or $A \in \mathbb{J}$.

5. Structured polar decomposition. The polar decomposition of a matrix $A \in \mathbb{C}^{n \times n}$ factors $A$ as a product $A=U H$, where $U$ is unitary and $H$ is Hermitian positive semidefinite. The Hermitian factor is always unique, whereas the unitary factor is unique only if $A$ is nonsingular [25]. When $A$ is real, so are $U$ and $H$. We begin by considering the polar factors of matrices belonging to automorphism groups, Lie algebras, and Jordan algebras associated with unitary scalar products, and investigate the extent to which these factors have similar structure. In section 5.2 the question of the structure of polar factors is addressed for automorphism groups of more general scalar products.

5.1. Unitary scalar products. Several conditions equivalent to a scalar product being unitary were given in section 2.3. Two of them are key to establishing the 
structure of the polar factors of matrices in $\mathbb{G}, \mathbb{L}$, and $\mathbb{J}$ : in a unitary scalar product space, adjoint preserves both unitarity and Hermitian positive definite structure (Definition 2.3, properties (b) and (c)).

\subsubsection{Automorphism groups.}

THEOREM 5.1. Suppose $\mathbb{G}$ is the automorphism group of a unitary scalar product, and $A \in \mathbb{G}$. Then in the polar decomposition $A=U H$ the factors $U$ and $H$ also belong to $\mathbb{G}$.

Proof. The matrix $A$ is nonsingular since $A \in \mathbb{G}$; thus $U$ and $H$ exist and are unique. We have

$$
A \in \mathbb{G} \Leftrightarrow A=A^{-\star}=(U H)^{-\star}=U^{-\star} H^{-\star} .
$$

By Definition 2.3, properties (b) and (c), $U^{\star}$ is unitary and $H^{\star}$ is Hermitian positive definite. Thus $A=U^{-\star} H^{-\star}$ exhibits another polar decomposition of $A$. Uniqueness of polar factors implies that $U=U^{-\star}$ and $H=H^{-\star}$; i.e., $U$ and $H$ are in $\mathbb{G}$.

Just as for the sign function, an alternate proof of this result can be given based on the existence of globally convergent structure-preserving iterations for the unitary polar factor of matrices in automorphism groups. Details about such iterations and their theoretical and numerical properties may be found in [23]. Connections between these polar factor iterations and other matrix iterations are explored and developed further in [24].

Note that Theorem 5.1 is known for some particular automorphism groups; for real symplectic matrices see [45], and for real pseudo-orthogonal matrices see [50].

5.1.2. Lie and Jordan algebras. Next we consider the polar factors of matrices in Lie and Jordan algebras associated with unitary scalar products. For a matrix $A$ in $\mathbb{L}$ or $\mathbb{J}$, when can we expect the polar factors of $A$ to be in $\mathbb{L}$ or $\mathbb{J}$ ? An added feature of this question that was not present for matrices in automorphism groups is that elements of $\mathbb{L}$ and $\mathbb{J}$ may be singular and thus have nonunique unitary polar factors. We look first at the unitary factor in the nonsingular case.

TheOrem 5.2. Suppose $A$ is nonsingular with polar decomposition $A=U H$. Let $\mathbb{S}$ denote either the Lie algebra $\mathbb{L}$ or the Jordan algebra $\mathbb{J}$ associated with a unitary scalar product. Then $A \in \mathbb{S} \Rightarrow U \in \mathbb{S}$.

Proof. We prove only the case when $\mathbb{S}=\mathbb{L}$; the argument when $\mathbb{S}=\mathbb{J}$ is almost identical. Since $A \in \mathbb{L}, A^{\star}=-A$ and

$$
A=U H \Rightarrow A^{\star}=H^{\star} U^{\star} \Rightarrow-A=H^{\star} U^{\star} \Rightarrow A=H^{\star}\left(-U^{\star}\right)=\widetilde{H} \widetilde{U} .
$$

This displays a "backwards" polar decomposition for $A$, since $H^{\star}$ is Hermitian positive definite and $U^{\star}$ is unitary. But for nonsingular matrices, the "forward" and "backward" unitary factors are equal [25]. Hence $U=\widetilde{U}=-U^{\star}$; i.e., $U \in \mathbb{L}$.

An alternative proof for Theorem 5.2 can be given using the Newton iteration $X_{k+1}=\frac{1}{2}\left(X_{k}+X_{k}^{-*}\right)$ for computing the unitary polar factor of the matrix $X_{0}$. For unitary scalar products it can be shown that this iteration is structure-preserving for all nonsingular matrices in $\mathbb{L}$ or $\mathbb{J}$. The global convergence of the iteration on nonsingular matrices then implies the desired result. More on Newton-like iterations can be found in [23] and [24].

To handle the singular case we need the following lemma.

Lemma 5.3. Suppose $\mathcal{W} \subseteq \mathbb{K}^{n \times n}$ is a (real or complex) subspace of matrices. Let $\operatorname{Inv}(\mathcal{W})$ denote the subset of invertible matrices in $\mathcal{W}$. Then either $\operatorname{Inv}(\mathcal{W})$ is empty, 
or it is an open dense subset of $\mathcal{W}$ whose complement $\mathcal{W} \backslash \operatorname{Inv}(\mathcal{W})$ is a set of measure zero in $\mathcal{W}$.

Proof. Let $\left\{W_{1}, W_{2}, \ldots, W_{\ell}\right\}$ be a basis for $\mathcal{W}$, so that every $X \in \mathcal{W}$ is uniquely expressed as $X=a_{1} W_{1}+\cdots+a_{\ell} W_{\ell}$. Then $p\left(a_{1}, a_{2}, \ldots, a_{\ell}\right)=\operatorname{det}\left(a_{1} W_{1}+\cdots+a_{\ell} W_{\ell}\right)$ is a polynomial in the unknowns $a_{1}, \ldots, a_{\ell}$ that distinguishes the singular elements from the nonsingular elements of $\mathcal{W}$; the zero set of $p$ in $\mathbb{K}^{\ell}$ can be identified with the set of singular matrices in $\mathcal{W}$. This zero set is not empty because $p(0,0, \ldots, 0)=0$; thus it is either all of $\mathcal{W}$ (if and only if $p \equiv 0$ ), or it is a nontrivial algebraic variety in $\mathcal{W}$. But nontrivial varieties are always closed and nowhere dense (in the usual Euclidean topology) and are of measure zero in $\mathcal{W}$.

With this lemma we can now deal with singular matrices in $\mathbb{L}$ or $\mathbb{J}$. It is possible that $\mathbb{L}$ or $\mathbb{J}$ may contain only singular matrices, as the lemma indicates (consider, for example, the Lie algebra of $n \times n$ skew-symmetric matrices for $n$ odd). In such a case, $\mathbb{L}$ (or $\mathbb{J})$ would contain no unitary matrices at all; this would be an insurmountable obstruction to the existence of a structured polar decomposition. Thus we must assume that $\mathbb{L}$ (or $\mathbb{J}$ ) contains at least one nonsingular matrix.

THEOREM 5.4. Let $\mathbb{S}$ denote either the Lie algebra $\mathbb{L}$ or the Jordan algebra $\mathbb{J}$ of a unitary scalar product, and suppose $A \in \mathbb{S}$ is a singular matrix. If $\mathbb{S}$ contains at least one nonsingular matrix, then there exists a polar decomposition $A=U H$ such that $U \in \mathbb{S} ;$ however, even this polar decomposition with $U \in \mathbb{S}$ need not be unique.

Proof. We consider only $A \in \mathbb{L}$, using a limit argument based on the already established result for the nonsingular case. The proof when $A \in \mathbb{J}$ is similar.

By assumption, $\mathbb{L}$ contains at least one nonsingular matrix; thus by Lemma 5.3 the nonsingular matrices are dense in $\mathbb{L}$. Let $A_{n} \rightarrow A$ with $A_{n} \in \mathbb{L}$ and $A_{n}$ nonsingular. By Theorem 5.2 each $A_{n}$ has a polar decomposition $A_{n}=U_{n} H_{n}$ with $U_{n} \in \mathbb{L}$. Since the unitary group is compact, we may pick a subsequence $U_{n_{k}}$ that converges to a unitary $U$; because $\mathbb{L}$ is closed, this $U$ is also in $\mathbb{L}$. Then the sequence $H_{n_{k}}=U_{n_{k}}^{-1} A_{n_{k}}$ converges, say to $H$. The set of Hermitian positive semidefinite matrices is also a closed set, and thus $H$ is Hermitian positive semidefinite. Then

$$
A=\lim A_{n}=\lim A_{n_{k}}=\lim U_{n_{k}} \cdot \lim H_{n_{k}}=U H
$$

displays a polar decomposition for $A$ with $U \in \mathbb{L}$. The nonuniqueness assertion is concretely demonstrated by Example 5.5.

Example 5.5. Consider the matrix

$$
A=\left[\begin{array}{ccccc}
0 & -2 & & \\
2 & 0 & & \\
& & 0 & 0 \\
& & 0 & 0
\end{array}\right]
$$

in the Lie algebra $\mathbb{L}$ of $4 \times 4$ real skew-symmetric matrices. For $A \in \mathbb{L}$ we have the polar decompositions

$$
\begin{aligned}
& A=U_{1} H=\left[\begin{array}{cccc}
0 & -1 & & \\
1 & 0 & & \\
& & 1 & 0 \\
& 0 & 1
\end{array}\right]\left[\begin{array}{llll}
2 & 0 & \\
0 & 2 & \\
& 0 & 0 \\
& 0 & 0
\end{array}\right] \text { with } U_{1} \notin \mathbb{L}, \\
& A=U_{2} H=\left[\begin{array}{cccc}
0 & -1 & & \\
1 & 0 & & \\
& 0 & -1 \\
& & 1 & 0
\end{array}\right]\left[\begin{array}{llll}
2 & 0 & \\
0 & 2 & \\
& 0 & 0 \\
& 0 & 0
\end{array}\right] \text { with } U_{2} \in \mathbb{L}, \\
& \text { and } A=U_{3} H=\left[\begin{array}{cccc}
0 & -1 & & \\
1 & 0 & & \\
& & 0 & 1 \\
& -1 & 0
\end{array}\right]\left[\begin{array}{llll}
2 & 0 & \\
0 & 2 & \\
& 0 & 0 \\
& 0 & 0
\end{array}\right] \text { with } U_{3} \in \mathbb{L} \text {, }
\end{aligned}
$$

illustrating the fact that a singular $A \in \mathbb{L}$ may have polar decompositions with the unitary factor in $\mathbb{L}$ or not in $\mathbb{L}$, as well as having more than one polar decomposi- 
tion with the unitary factor in $\mathbb{L}$. Similar examples may be constructed for singular matrices in the Jordan algebra of $4 \times 4$ real symmetric matrices.

What about the structure of the Hermitian factor $H$ when $A$ is in $\mathbb{L}$ or $\mathbb{J}$ ? Though $H$ is always unique, regardless of whether $A$ is singular or nonsingular, in general $H$ does not seem to have any additional structure beyond Hermitian positive semidefiniteness. However, there is one special situation where $H$ does have extra structure.

THEOREm 5.6. Suppose $A$ is in $\mathbb{L}$ or $\mathbb{J}$ of a unitary scalar product, and $H$ is the Hermitian positive semidefinite polar factor of $A$. Then $H \in \mathbb{J}$ if and only if $A$ is normal, i.e., $A^{*} A=A A^{*}$.

Proof. We consider only the case $A \in \mathbb{L}$, since the proof for $A \in \mathbb{J}$ is almost identical.

$(\Rightarrow)$ : By Lemma 2.2(b), $H=\left(A^{*} A\right)^{1 / 2} \in \mathbb{J} \Rightarrow A^{*} A \in \mathbb{J}$; i.e., $\left(A^{*} A\right)^{\star}=A^{*} A$. So then $A^{*} A=\left(A^{*} A\right)^{\star}=A^{\star}\left(A^{*}\right)^{\star}=A^{\star}\left(A^{\star}\right)^{*}$, since the stars commute in any unitary scalar product space (see Definition $2.3(\mathrm{a})$ ). But $A \in \mathbb{L}$ means $A^{\star}=-A$, and therefore $A^{*} A=A^{\star}\left(A^{\star}\right)^{*}=(-A)(-A)^{*}=A A^{*}$. Thus $A$ is normal.

$(\Leftarrow)$ : By Proposition 2.5, $A \in \mathbb{L} \Rightarrow A^{*} \in \mathbb{L}$. Then since $A$ is normal, we have $A^{*} A \in \mathbb{J}$ by Lemma 2.2(e). By Theorem 3.4 we then have $H=\left(A^{*} A\right)^{1 / 2} \in \mathbb{J}$.

5.2. General scalar products. We now turn to the question of the polar factors of matrices in the automorphism group of a general scalar product. The results of section 3 on structured principal square roots allow us to give conditions under which an individual $A \in \mathbb{G}$ has both of its polar factors in $\mathbb{G}$, without making any global assumption about the scalar product or the automorphism group.

THEOREM 5.7. Let $\mathbb{G}$ be the automorphism group of an arbitrary scalar product, and suppose $A \in \mathbb{G}$. Then the following are equivalent:

(a) $\left(A^{*}\right)^{\star}=\left(A^{\star}\right)^{*}$ (i.e., "the stars commute on $A^{\prime}$ ).

(b) $A^{*} \in \mathbb{G}$.

(c) A has a structured polar decomposition; i.e., $A=U H$ with $U, H \in \mathbb{G}$.

Proof. $(\mathrm{a} \Rightarrow \mathrm{b}): A^{\star}=A^{-1} \Rightarrow\left(A^{\star}\right)^{*}=\left(A^{-1}\right)^{*} \Rightarrow\left(A^{*}\right)^{\star}=\left(A^{*}\right)^{-1} \Rightarrow A^{*} \in \mathbb{G}$.

$(\mathrm{b} \Rightarrow \mathrm{c})$ : Suppose $A=U H$ is the polar decomposition of $A$. Since $A^{*} \in \mathbb{G}$ we have $A^{*} A=H^{2} \in \mathbb{G}$, and thus $H=\left(H^{2}\right)^{1 / 2} \in \mathbb{G}$ by Theorem 3.2. But then $U=$ $A H^{-1} \in \mathbb{G}$ because $\mathbb{G}$ is a group, and thus $A$ has a structured polar decomposition.

$(\mathrm{c} \Rightarrow \mathrm{a})$ : On one side, $\left(A^{*}\right)^{\star}=\left((U H)^{*}\right)^{\star}=\left(H U^{-1}\right)^{\star}=U^{-\star} H^{\star}=U H^{-1}$, while on the other side, $\left(A^{\star}\right)^{*}=\left((U H)^{\star}\right)^{*}=\left(H^{-1} U^{-1}\right)^{*}=U^{-*} H^{-*}=U H^{-1}$.

In what follows, for any scalar product, we say that the automorphism group $\mathbb{G}$ "has structured polar decompositions" if for every $A \in \mathbb{G}$ we have $U, H \in \mathbb{G}$, where $A=U H$ is the polar decomposition of $A$.

COROLlARY 5.8. An automorphism group $\mathbb{G}$ has structured polar decompositions if and only if $\mathbb{G}$ is closed under conjugate transpose.

The next results identify two classes of scalar products whose automorphism groups have structured polar decompositions. Characterizing the set of all scalar products whose automorphism groups have structured polar decompositions is still an open question.

COROLLARY 5.9. The automorphism group of any unitary scalar product has structured polar decompositions.

Proof. This has already been proved in Theorem 5.1. But it is also a consequence of Corollary 5.8, together with the fact that $\mathbb{G}$ is closed under conjugate transpose when the scalar product is unitary (Proposition 2.5).

Corollary 5.10 extends a result of Horn, Merino, and Serre [28], who considered scalar products $\langle\cdot, \cdot\rangle_{\mathrm{M}}$ defined by real $M$ with $M^{2}= \pm I$. In this corollary, note that 
$M$ may be complex in the sesquilinear case.

COROLlary 5.10. Let $\langle\cdot, \cdot\rangle_{\mathrm{M}}$ be a scalar product on $\mathbb{K}^{n}$ such that $M^{2}=\alpha I$ for some nonzero $\alpha \in \mathbb{K}$. In the complex bilinear case, further assume that $M$ is real. Then the associated automorphism group $\mathbb{G}$ has structured polar decompositions.

Proof. From (2.2) it follows that

$$
\left(A^{*}\right)^{\star}= \begin{cases}M^{-1} \bar{A} M, & \text { bilinear forms, } \\ M^{-1} A M, & \text { sesquilinear forms. }\end{cases}
$$

Observe that $M^{2}=\alpha I$ implies

$$
M^{-1} A M=M A M^{-1} \text { for all } A \in \mathbb{K}^{n \times n} .
$$

Our strategy will be to show that $\mathbb{G}$ is closed under conjugate transpose.

Complex sesquilinear forms: $A \in \mathbb{G} \Rightarrow A^{\star} A=I \Rightarrow M^{-1} A^{*} M A=I$. Thus $M^{-1} A^{*}$ and $M A$ are inverses and hence commute, giving us $M A M^{-1} A^{*}=I$. Using (5.2), this becomes $M^{-1} A M A^{*}=I$, or $\left(A^{*}\right)^{\star} A^{*}=I$ by (5.1). Hence $A^{*} \in \mathbb{G}$.

Real or complex bilinear forms with $M$ real: Proceeding along similar lines we obtain $A \in \mathbb{G} \Rightarrow M^{-1} A M A^{T}=I$. Taking conjugates and using the fact that $M$ is real gives $M^{-1} \bar{A} M A^{*}=I$, or $\left(A^{*}\right)^{\star} A^{*}=I$, and thus $A^{*} \in \mathbb{G}$.

The example below illustrates that for complex bilinear forms $\langle\cdot, \cdot\rangle_{\mathrm{M}}$, the hypothesis $M^{2}=\alpha I$ by itself is not sufficient to guarantee that $\mathbb{G}$ has structured polar decompositions. Hence the necessity for some additional hypothesis like $M$ is real in Corollary 5.10.

Example 5.11. Consider the bilinear form on $\mathbb{C}^{2}$ defined by $M=\left[\begin{array}{cc}1 & i \\ 0 & -1\end{array}\right]$. Note that $M=\left[\begin{array}{cc}1 & \beta \\ 0 & -1\end{array}\right]$ satisfies $M^{2}=I$ for any $\beta \in \mathbb{C}$. A direct calculation shows that $A \in \mathbb{G}$ if and only if $\mathrm{A}$ is of the form $\left[\begin{array}{cc}a & b \\ b & a+i b\end{array}\right]$ with $a, b \in \mathbb{C}$ and $\operatorname{det} A=+1$. Hence $\mathbb{G}$ is far from being closed under conjugate transpose. Indeed, both $A$ and $A^{*}$ belong to $\mathbb{G}$ only when $A= \pm I$; therefore these are the only elements of $\mathbb{G}$ with structured polar decompositions.

6. Generalized polar decomposition. In this section we look at a factorization that is somewhat different in nature from all the others considered in this paper. In other sections we take as starting point a matrix $A$ in either $\mathbb{G}, \mathbb{L}$, or $\mathbb{J}$ of a scalar product space and then study the extent to which some standard factorization produces factors of $A$ that are also in $\mathbb{G}, \mathbb{L}$, or $\mathbb{J}$. Here we begin instead with a general matrix $A \in \mathbb{K}^{n \times n}$ and seek to decompose it as $A=W S$, where $W$ is in the automorphism group and $S$ is in the Jordan algebra of some scalar product on $\mathbb{K}^{n}$. This notion generalizes the standard polar decomposition, where the scalar product is the standard inner product $\langle x, y\rangle=x^{*} y$ on $\mathbb{C}^{n}$, the automorphism group is the set of unitary matrices, and the Jordan algebra is the set of Hermitian matrices. Since there are scalar products for which the spectra of matrices in $\mathbb{J}$ can be arbitrary finite subsets of $\mathbb{C}$ (e.g., $\langle x, y\rangle=x^{T} y$ on $\mathbb{C}^{n}$, with $\mathbb{J}$ the set of complex symmetric matrices), it is not immediately clear what might constitute an appropriate replacement for the positive semidefiniteness condition on the Hermitian factor in the usual polar decomposition. We will return to this issue after briefly surveying some of the literature on this factorization.

The idea of generalizing the polar decomposition in this way is not new; it has been studied independently by several groups of authors using various notation and terminology, and in various contexts. An extensive body of work on this topic is due to Bolshakov, Reichstein, and others, starting with [5], [6], and [7], and continuing 
through the recent [33] and [42]. In these papers the basic setting is a scalar product space defined by a Hermitian sesquilinear form on $\mathbb{C}^{n}$, termed an "indefinite inner (or scalar) product space." Questions of existence of factorizations $A=W S(W \in$ $\mathbb{G}, S \in \mathbb{J}$ ) are considered with $S$ satisfying various types of extra conditions, and even no extra condition at all, with technical emphasis on the more difficult case when $A$ is singular. Kintzel [33] studies the doubly structured existence question: Given two scalar products with associated groups $\mathbb{G}_{1}, \mathbb{G}_{2}$ and Jordan algebras $\mathbb{J}_{1}, \mathbb{J}_{2}$, when can a matrix $A$ be factored as $A=W S$ with $W \in \mathbb{G}_{1} \cap \mathbb{G}_{2}$ and $S \in \mathbb{J}_{1} \cap J_{2}$ ? Another recurring theme in these papers is the characterization of special classes of matrices (e.g., matrices that are normal with respect to the given scalar product) for which every $A$ in the class can be factored as $A=W S$. Complementary to this work are papers of Kaplansky [31] and Ikramov [29]; Kaplansky considers symmetric bilinear forms on $\mathbb{K}^{n}$ for algebraically closed fields $\mathbb{K}$, while Ikramov treats only the symplectic bilinear form on $\mathbb{C}^{2 n}$.

In [27], Horn and Merino investigate a matrix factorization which they term the $\Phi_{S}$-polar decomposition. Here the setting is not a scalar product space but rather the complex matrices $\mathbb{C}^{n \times n}$ equipped with a linear operator $\Phi_{S}(A):=S A^{T} S^{-1}$ for some fixed nonsingular symmetric or skew-symmetric $S \in \mathbb{C}^{n \times n}$. Matrices $A$ such that $\Phi_{S}(A)=A$ are called $\Phi_{S}$-symmetric, and matrices $A$ such that $\Phi_{S}(A)=A^{-1}$ are $\Phi_{S}$-orthogonal. Then $A$ is said to have a $\Phi_{S}$-polar decomposition if $A=X Y$ for some $\Phi_{S}$-orthogonal $X$ and $\Phi_{S}$-symmetric $Y$. This way of generalizing the usual polar decomposition (on $\mathbb{R}^{n \times n}$ ) is easily seen to be a special case of the generalized polar decomposition in scalar product spaces described above; if $M=S^{-1}$ is used to define a bilinear form $\langle x, y\rangle_{\mathrm{M}}=x^{T} M y$ on $\mathbb{C}^{n}$, then $\Phi_{S}(A)$ is the same as the adjoint $A^{\star}$ with respect to this scalar product, and $\mathbb{G}=\left\{\Phi_{S}\right.$-orthogonals $\}$ while $\mathbb{J}=\left\{\Phi_{S}\right.$-symmetrics $\}$. Horn and Merino show that for any such $\Phi_{S}$, every nonsingular $A$ has a $\Phi_{S}$-polar decomposition, and also give necessary and sufficient conditions for a general $A$ to have a $\Phi_{S}$-polar decomposition.

Yet a different approach to this factorization uses methods from Lie theory. Here the setting is a Lie group (or Lie semigroup) $G$ equipped with an involutive homomorphism $\sigma: G \rightarrow G$; i.e., $\sigma^{2}=$ id but $\sigma \neq$ id. A generalized polar decomposition (or Ol'shanskii decomposition) of an element $z \in G$ in this setting is a factorization $z=x y$, where $x$ is an element of the fixed point set $G^{\sigma}=\{x \in G: \sigma(x)=x\}$ and $y$ is an element of the anti-fixed point set $G_{\sigma}=\left\{y \in G: \sigma(y)=y^{-1}\right\}$. See [35] for a development of the basic theory of this decomposition. A fundamental example is the group $G=G L(n, \mathbb{R})$ of all nonsingular real $n \times n$ matrices with the map $\sigma(A)=A^{-T}$. In this example $G^{\sigma}$ is the group of real orthogonal matrices and $G_{\sigma}$ is the set of all nonsingular symmetric matrices, and thus we are back to the usual notion of polar decomposition. Indeed, for any orthosymmetric scalar product on $\mathbb{K}^{n}$, the map $\sigma(A)=A^{-\star}$ is an involutive homomorphism on the group $G=G L(n, \mathbb{K})$, with $G^{\sigma}=\mathbb{G}$ and $G_{\sigma}$ the set of all nonsingular elements of $\mathbb{J}$.

A disadvantage of this Lie group setting is that singular matrices are not considered at all; however, there are compensating advantages. The most important advantage is that many more examples than just the scalar product space notion of generalized polar decomposition can be encompassed in a unified development, including both matrix groups equipped with involutions $\sigma$ that do not arise from any scalar product structure, as well as nonmatrix Lie groups associated with the study of differential equations. For more on such examples and a further development of the theory, see [46]. This Lie group notion of generalized polar decomposition has also recently been exploited in [51] and [30] to develop new structure-preserving algorithms 
for computing the matrix exponential.

We return now to consider the question of an appropriate generalization for the positive definiteness condition on the self-adjoint (Hermitian) factor in the usual polar decomposition. Several such replacements have been suggested in the literature, but to us the simplest approach is to restrict the eigenvalues of the self-adjoint factor $S \in \mathbb{J}$ to some region of the complex plane containing the positive real axis, so as to compatibly generalize the usual positive definiteness condition. The most natural such region for the eigenvalues of $S$ is the open right half-plane; this eigenvalue condition is concisely expressed as $\operatorname{sign}(S)=I$. Bhatia [3] and Kintzel [33] use this condition: Bhatia only for the bilinear form $\langle x, y\rangle=x^{T} y$ on $\mathbb{C}^{n}$, and Kintzel for Hermitian sesquilinear and real symmetric bilinear forms.

Further justifying the choice of this particular condition is that it gives a generalized polar decomposition (GPD) that is strongly connected with the matrix sign function and the principal square root, both of which are well known to be computable by matrix iterations (see [21] and [32]). This connection is elaborated on and expanded in [24] to show how large families of matrix iterations for the matrix sign function and the principal square root are related to each other and to iterations for the GPD with the $\operatorname{sign}(S)=I$ condition (see also [33] for one particular iteration from this family). One may reasonably view this GPD as the "computable" generalized polar decomposition, and thus we adopt the following definition, both for this paper and for [24], where iterations to compute this GPD are derived and analyzed.

DEFINITION 6.1 ("computable" GPD). Suppose $\mathbb{K}^{n}$ is a scalar product space with automorphism group $\mathbb{G}$ and Jordan algebra $\mathbb{J}$. Then for a matrix $A \in \mathbb{K}^{n \times n}$, $a$ generalized polar decomposition (GPD) with respect to this scalar product is a factorization $A=W S$, where $W \in \mathbb{G}, S \in \mathbb{J}$, and $\operatorname{sign}(S)=I$.

Although the condition $\operatorname{sign}(S)=I$ immediately restricts us to considering only nonsingular $A$, it also leads to a connection between this notion of a GPD and the principal square root, as will be seen in the proof of Theorem 6.2. This theorem deals with the existence and uniqueness of a ("computable") GPD for an individual matrix, with respect to an arbitrary scalar product.

THEOREM 6.2. Let $\mathbb{K}^{n}$ be an arbitrary scalar product space. Then a matrix $A \in \mathbb{K}^{n \times n}$ has a GPD with respect to this scalar product if and only if $\left(A^{\star}\right)^{\star}=A$ and $A^{\star} A$ has no eigenvalues on $\mathbb{R}^{-}:=\{x \in \mathbb{R}: x \leq 0\}$. When such a factorization exists it is unique.

Proof. $(\Rightarrow)$ Note first that if the factorization exists, then

$$
\left(A^{\star}\right)^{\star}=\left(S^{\star} W^{\star}\right)^{\star}=\left(S W^{-1}\right)^{\star}=W^{-\star} S^{\star}=\left(W^{-1}\right)^{-1} S=W S=A .
$$

Also we must have

$$
A^{\star} A=S^{\star} W^{\star} W S=S^{\star} S=S^{2} .
$$

But if $\operatorname{sign}(S)=I$ is to hold, then the only possible choice for $S$ is the principal square root $S=\left(A^{\star} A\right)^{1 / 2}$, and this square root exists only if $A^{\star} A$ has no eigenvalues on $\mathbb{R}^{-}$.

$(\Leftarrow)$ By hypothesis, $A^{\star} A$ has a principal square root. Letting $S=\left(A^{\star} A\right)^{1 / 2}$, the condition $\operatorname{sign}(S)=I$ is automatically satisfied. It remains to show that $S^{\star}=S$. Using Lemma 3.1(b) together with the assumption that $\left(A^{\star}\right)^{\star}=A$, we have

$$
S^{\star}=\left(\left(A^{\star} A\right)^{1 / 2}\right)^{\star}=\left(\left(A^{\star} A\right)^{\star}\right)^{1 / 2}=\left(A^{\star} A\right)^{1 / 2}=S .
$$

Finally, since $S^{2}=A^{\star} A$, the uniquely defined matrix $W=A S^{-1}$ satisfies

$$
W^{\star} W=\left(A S^{-1}\right)^{\star}\left(A S^{-1}\right)=S^{-\star}\left(A^{\star} A\right) S^{-1}=S^{-1}\left(S^{2}\right) S^{-1}=I,
$$


and thus $W \in \mathbb{G}$. $\quad$ 口

A matrix factorization is most useful in practice if it exists for a large set of matrices, certainly for at least an open subset of $\mathbb{K}^{n \times n}$. With this in mind, we examine the two conditions in Theorem 6.2 to see which scalar products are capable of supporting a generalized polar decomposition (with or without the condition $\operatorname{sign}(S)=I$ ) that exists on some open subset of matrices.

For any scalar product the matrix $A=I$ produces an $A^{\star} A$ that has no eigenvalues on $\mathbb{R}^{-}$. The continuity of the eigenvalues of $A^{\star} A$ under perturbation of $A$ then implies that there will always be some (perhaps very large) open neighborhood of the identity in $\mathbb{K}^{n \times n}$ where the eigenvalue condition $\sigma\left(A^{\star} A\right) \cap \mathbb{R}^{-}=\emptyset$ holds for all $A$ in the neighborhood. Thus the eigenvalue condition on $A^{\star} A$ in Theorem 6.2 puts no constraint on which scalar products can support a useful generalized polar decomposition.

Turning next to the involutory property $\left(A^{\star}\right)^{\star}=A$, we will see that the situation is somewhat different. For a general scalar product we can always expect there to be a large set of matrices for which $\left(A^{\star}\right)^{\star}=A$ holds, as the next result shows, but this set need not always contain an open set.

LEMma 6.3. For a scalar product space $\mathbb{K}^{n},\left(A^{\star}\right)^{\star}=A$ holds for all $A$ in the $\mathbb{K}$-subalgebra of $n \times n$ matrices generated by $\mathbb{G} \cup \mathbb{L} \cup \mathbb{J}$.

Proof. The straightforward proof is omitted.

However, from property (e') in Definition 2.4 we know that the involutory property of adjoint holds in some open set of matrices if and only if the scalar product is orthosymmetric. Thus we are led to the following theorem.

TheOREM 6.4. Let $\langle\cdot, \cdot\rangle$ be a scalar product on $\mathbb{K}^{n}$. Then there exists some open ${ }^{2}$ subset $\mathcal{U} \subseteq \mathbb{K}^{n \times n}$ of matrices such that every $A \in \mathcal{U}$ can be factored as $A=W S$ with $W \in \mathbb{G}$ and $S \in \mathbb{J}$ if and only if the scalar product $\langle\cdot, \cdot\rangle$ is orthosymmetric. (Note that we make no assumption here on $S$ other than $S \in \mathbb{J}$, nor is the factorization $A=W S$ assumed to be unique. See Theorem A.5 for a further strengthening of this result.)

Proof. $(\Rightarrow)$ For all $A \in \mathcal{U}$ we have

$$
\left(A^{\star}\right)^{\star}=\left(S^{\star} W^{\star}\right)^{\star}=\left(S W^{-1}\right)^{\star}=W^{-\star} S^{\star}=\left(W^{-1}\right)^{-1} S=W S=A .
$$

Thus by Lemma A.2(e) and Theorem A.5(a') we know that $\langle\cdot, \cdot\rangle$ must be orthosymmetric.

$(\Leftarrow)$ From Theorem A.5(a), Theorem 6.2, and the earlier discussion of the spectrum of $A^{\star} A$ for $A$ near $I$, we see that there is some open neighborhood of $I$ in which every matrix $A$ has a GPD.

Theorem 6.4 provides a compelling reason why one might choose to consider only orthosymmetric scalar products when studying generalized polar decompositions of any kind, even those without the condition $\operatorname{sign}(S)=I$, or those that lack uniqueness of the factorization. Furthermore, this result also clarifies the mathematical necessity of the choices of scalar product made in previous work, which might otherwise appear to be unnecessarily restrictive or motivated only by a particular application. For orthosymmetric scalar products, then, we have the following simplification of Theorem 6.2.

COROLlaRY 6.5. For $\mathbb{K}^{n}$ equipped with an orthosymmetric scalar product, a matrix $A \in \mathbb{K}^{n \times n}$ has a GPD if and only if $A^{\star} A$ has no eigenvalues on $\mathbb{R}^{-}$. Whenever this GPD exists it is unique.

\footnotetext{
${ }^{2}$ This is with respect to the standard Euclidean topology on $\mathbb{K}^{n \times n}$.
} 

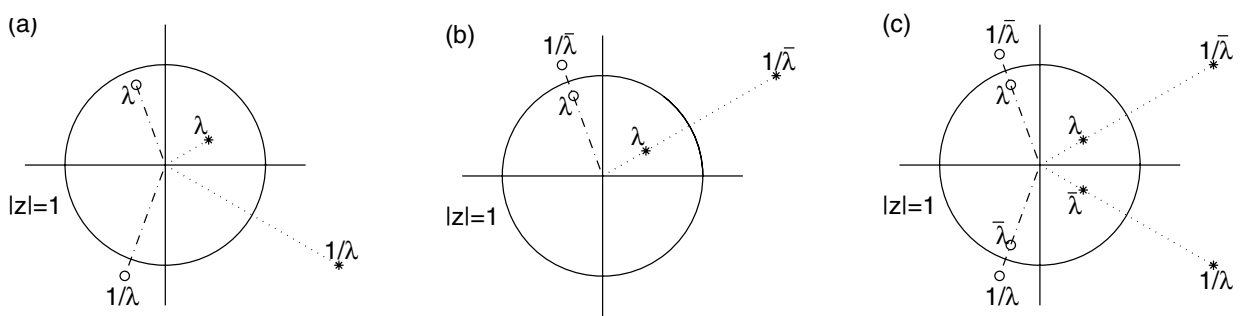

FIG. 7.1. Eigenvalue pairings relative to the unit circle in (a) general bilinear, (b) sesquilinear, and (c) real bilinear cases.

7. Eigenvalue and eigenvector structure. Even when no restrictions are imposed on the scalar product, matrices in the corresponding automorphism group and Lie and Jordan algebras have structure in their eigenvalues and eigenvectors. Some of this structure has been observed and exploited before, but usually in specific cases. In the context of a fixed scalar product or a restricted class of scalar products, the pursuit of spectral decompositions, Jordan canonical forms, and other condensed forms under structure-preserving similarities or general similarities has been the focus of intense study. The literature on this subject is extensive; see, for example, [1], [2], [12], [15], [18], [36], [38], [40], [41], [44], [43], and the references therein.

In this section we give a simple, unified presentation of the common eigenstructure shared by matrices in $\mathbb{G}, \mathbb{L}$, or $\mathbb{J}$ associated with an arbitrary scalar product. Our aim is to shed light on the extent to which the structure of eigenvalues and eigenvectors is independent of the particular scalar product defining the classes $\mathbb{G}, \mathbb{L}$, and $\mathbb{J}$.

7.1. Eigenvalues of automorphisms. The determinant and eigenvalues that are possible for matrices in automorphism groups are restricted.

Proposition 7.1. Let $A \in \mathbb{G}$. Then $\operatorname{det} A= \pm 1$ for any bilinear form, while for sesquilinear forms $|\operatorname{det} A|=1$.

Proof. For bilinear forms, $A \in \mathbb{G} \Rightarrow A^{\star} A=I \Rightarrow M^{-1} A^{T} M A=I \Rightarrow(\operatorname{det} A)^{2}=$ $1 \Rightarrow \operatorname{det} A= \pm 1$, while for sesquilinear forms, $A^{\star} A=I \Rightarrow M^{-1} A^{*} M A=I \Rightarrow$ $\overline{\operatorname{det} A} \operatorname{det} A=1 \Rightarrow|\operatorname{det} A|=1$.

In some automorphism groups, the determinant can be even more restricted; for example, real and complex symplectic matrices have only +1 determinant, and -1 is never realized. For a collection of proofs of this result, see [37].

Next we turn to the pairing structure found in the eigenvalues of matrices in $\mathbb{G}$.

Theorem 7.2. Let $A \in \mathbb{G}$. The eigenvalues of $A$ come in pairs $\lambda$ and $1 / \lambda$ for bilinear forms and in pairs $\lambda$ and $1 / \bar{\lambda}$ for sesquilinear forms. In both cases these pairs have the same Jordan structure and hence the same algebraic and geometric multiplicities.

Proof. From Lemma 2.1 we have $A^{-1}=A^{\star} \sim A$ for bilinear forms, whereas $A^{-1}=A^{\star} \sim \bar{A}$ for sesquilinear forms. The result is now immediate.

These eigenvalue pairings are illustrated in Figure 7.1(a) and (b). Instances of Theorem 7.2 for specific automorphism groups can be found, for example, in [8] and [22].

The automorphism group of a real bilinear form can be viewed as a restriction to real matrices of either the automorphism group of a complex bilinear form or the automorphism group of a complex sesquilinear form. Hence both eigenvalue structure results apply to real automorphisms, yielding the following corollary. 
Corollary 7.3. Let $A \in \mathbb{G}$, where $\mathbb{G}$ is the automorphism group of a real bilinear form. Then the eigenvalues of $A$ come in quartets $\lambda, 1 / \lambda, \bar{\lambda}, 1 / \bar{\lambda}$. Figure 7.1(c) depicts such quartets of eigenvalues relative to the unit circle in $\mathbb{C}$.

7.2. Characteristic polynomials of automorphisms. We next describe a nice property of the characteristic polynomials of matrices in automorphism groups. For a polynomial $p(x)=a_{n} x^{n}+a_{n-1} x^{n-1}+\cdots+a_{1} x+a_{0}$ of degree $n$ (i.e., $a_{n} \neq 0$ ), we define the "reversal of $p(x)$ " to be the polynomial $\operatorname{rev} p(x)=a_{0} x^{n}+a_{1} x^{n-1}+$ $\cdots+a_{n-1} x+a_{n}$, that is, the polynomial $p$ with its coefficients in reverse order. It is easy to see that

$$
\operatorname{rev} p(x)=x^{n} p(1 / x)
$$

The "conjugate of $p(x)$ " is the polynomial $\bar{p}(x)=\bar{a}_{n} x^{n}+\bar{a}_{n-1} x^{n-1}+\cdots+\bar{a}_{1} x+\bar{a}_{0}$.

DeFinition 7.4. We say that $p(x)$ is

- palindromic if $\operatorname{rev} p(x)=p(x)$,

- anti-palindromic if $\operatorname{rev} p(x)=-p(x)$,

- conjugate palindromic if $\operatorname{rev} p(x)=\bar{p}(x)$,

- conjugate anti-palindromic if $\operatorname{rev} p(x)=-\bar{p}(x)$.

Theorem 7.5. Suppose $A \in \mathbb{G}$. Let $p_{A}(x)=\operatorname{det}(x I-A)$ denote the characteristic polynomial of $A$, and let $s:=(-1)^{n} \operatorname{det}(A)$.

(a) For bilinear forms, $s= \pm 1 ; p_{A}(x)$ is palindromic if $s=1$ and anti-palindromic if $s=-1$.

(b) For sesquilinear forms, $|s|=1$, and $q_{A}(x):=\overline{s^{1 / 2}} p_{A}(x)$ is conjugate palindromic. Thus $p_{A}(x)$ is a scalar multiple of a conjugate palindromic polynomial.

Proof. These results can be derived as consequences of the eigenvalue structure proved in Theorem 7.2, but we include a more direct proof here.

(a) As shown in the proof of Theorem 7.2, we have $A \sim A^{-1}$. From Proposition 7.1 we know that $\operatorname{det} A= \pm 1$. Thus,

$$
\begin{aligned}
p_{A}(x) & =p_{A^{-1}}(x)=(\operatorname{det} A)^{2} \operatorname{det}\left(x I-A^{-1}\right) \\
& =(\operatorname{det} A) \operatorname{det}(x A-I)=(\operatorname{det} A) \operatorname{det}\left((-x I)\left(x^{-1} I-A\right)\right) \\
& =(\operatorname{det} A)(-x)^{n} p_{A}\left(x^{-1}\right)=(-1)^{n}(\operatorname{det} A) \operatorname{rev} p_{A}(x) \\
& =( \pm 1) \operatorname{rev} p_{A}(x) .
\end{aligned}
$$

Hence $p_{A}(x)$ is either palindromic or anti-palindromic, depending on the sign of $s=$ $(-1)^{n} \operatorname{det}(A)$.

(b) In this case we have $A \sim \bar{A}^{-1}$ and $(\operatorname{det} A)(\overline{\operatorname{det} A})=1$. Since $\operatorname{det} A$ can now have complex values other than \pm 1 , it will not be as straightforward to see palindromicity here. We have

$$
\begin{aligned}
p_{A}(x) & =p_{\bar{A}^{-1}}(x)=(\operatorname{det} A)(\overline{\operatorname{det} A}) \operatorname{det}\left(x I-\bar{A}^{-1}\right) \\
& =(\operatorname{det} A) \operatorname{det}(x \bar{A}-I)=(\operatorname{det} A) \operatorname{det}\left((-x I)\left(x^{-1} I-\bar{A}\right)\right) \\
& =(\operatorname{det} A)(-x)^{n} p_{\bar{A}}\left(x^{-1}\right)=(-1)^{n}(\operatorname{det} A) \operatorname{rev} \bar{p}_{A}(x) .
\end{aligned}
$$

So if $\operatorname{det} A= \pm 1$, then $p_{A}(x)$ is already conjugate palindromic or conjugate antipalindromic. But in general $s:=(-1)^{n} \operatorname{det} A=e^{i \theta}$, and $p_{A}(x)$ itself will not usually be conjugate (anti)palindromic. However, it is always possible to "symmetrize" $p_{A}(x)$ by 
an appropriate complex scalar multiple that makes the resulting polynomial conjugate palindromic. Define $q_{A}(x)=\overline{s^{1 / 2}} p_{A}(x)=e^{-i \theta / 2} p_{A}(x)$. Then using (7.1) we get

$$
q_{A}(x)=e^{-i \theta / 2} e^{i \theta} \operatorname{rev} \bar{p}_{A}(x)=e^{i \theta / 2} \operatorname{rev} \bar{p}_{A}(x)=\operatorname{rev} \bar{q}_{A}(x) .
$$

Thus $q_{A}(x)$ is conjugate palindromic.

7.3. Eigenvalues of matrices in Lie and Jordan algebras. Analogous to the situation for automorphism groups, the relationship between matrices in $\mathbb{L}$ or $\mathbb{J}$ and their adjoints also leads to a pairing of eigenvalues and their corresponding Jordan blocks, as the next result shows.

TheOrem 7.6. Let $A \in \mathbb{L}$ or $A \in \mathbb{J}$. Then the eigenvalues of $A$ occur in pairs as shown below, with the same Jordan structure for each eigenvalue in a pair.

\begin{tabular}{c|cc}
\hline & Bilinear & Sesquilinear \\
\hline$A \in \mathbb{L}$ & $\lambda,-\lambda$ & $\lambda,-\bar{\lambda}$ \\
$A \in \mathbb{J}$ & "no pairing" & $\lambda, \bar{\lambda}$ \\
\hline
\end{tabular}

Proof. When $A \in \mathbb{L}$, then $A^{\star}=-A$ by definition. Lemma 2.1 now implies $A \sim-A$ in the bilinear case and $A \sim-\bar{A}$ in the sesquilinear case, thus forcing eigenvalue pairings $\lambda,-\lambda$ and $\lambda,-\bar{\lambda}$, respectively, as well as the identical Jordan block structure for each eigenvalue in a pair.

On the other hand, when $A \in \mathbb{J}, A^{\star}=A$. In the bilinear case Lemma 2.1 puts no constraints on the eigenvalues. However, in the sesquilinear case we get $A \sim \bar{A}$, forcing the pairing $\lambda, \bar{\lambda}$ as eigenvalues of $A$ with the same Jordan structure.

It is well known that any finite subset of $\mathbb{C}$ can be realized as the spectrum of a complex symmetric matrix [25, Thm. 4.4.9]. Thus there can be no eigenvalue structure property that holds in general for Jordan algebras of all bilinear forms; this explains the "no pairing" entry in the table in Theorem 7.6. On the other hand, for certain special classes of $\mathbb{L}$ or $\mathbb{J}$ there may be additional structure in the eigenvalues for reasons other than those considered in Theorem 7.6. For example, it is known that the eigenvalues of any real or complex skew-Hamiltonian matrix all have even multiplicity [16], [17], [29]. This is a special case of the following result.

Proposition 7.7. Let $\mathbb{J}$ be the Jordan algebra of any skew-symmetric bilinear form on $\mathbb{K}^{n}$. Then for any $A \in \mathbb{J}$, the eigenvalues of $A$ all have even multiplicity. Moreover, for any $m>0$ and eigenvalue $\lambda$, the number of $m \times m$ Jordan blocks corresponding to $\lambda$ in the Jordan form for $A$ is even.

Proof. Suppose $M$ is the $n \times n$ matrix defining the given skew-symmetric bilinear form; note that $n$ is even since $M$ is nonsingular. Then $A \in \mathbb{J} \Rightarrow A^{\star}=A \Rightarrow$ $M^{-1} A^{T} M=A \Rightarrow A^{T} M=M A \Rightarrow(M A)^{T}=-M A$. Thus $A=M^{-1} \cdot(M A)$ expresses $A$ as the product of two skew-symmetric matrices, the first of which is nonsingular. But any such product has an even number of $m \times m$ Jordan blocks for any eigenvalue $\lambda$, by Propositions 3 and 4 in [29]. The even multiplicity of all eigenvalues of $A$ now follows immediately.

7.4. Eigenvector structure. The eigenvectors of matrices in $\mathbb{G}, \mathbb{L}$, or $\mathbb{J}$ cannot in general be arbitrarily chosen from $\mathbb{K}^{n}$, nor can they be arbitrarily matched up with scalars in $\mathbb{K}$ to form eigenpairs. In this section we will see what these restrictions on eigenvectors are and how they can sometimes feed back to put extra constraints on the spectra of structured matrices.

To concisely describe the relationships among the eigenvectors and their corresponding eigenvalues, we introduce various eigenvalue "pairing functions" $\wp$ to sum- 
marize the eigenvalue structure results of Theorems 7.2 and 7.6. The function $\wp$ depends on the type of structured matrix under consideration, and associates to a scalar $\lambda$ the eigenvalue "partner" appropriate to that structure.

\begin{tabular}{c|c|c|c}
\hline Scalar product & $\mathbb{G}$ & $\mathbb{L}$ & $\mathbb{J}$ \\
\hline Bilinear & $\wp(\lambda):=1 / \lambda$ & $\wp(\lambda):=-\lambda$ & "no pairing" \\
Sesquilinear & $\wp(\lambda):=1 / \bar{\lambda}$ & $\wp(\lambda):=-\bar{\lambda}$ & $\wp(\lambda):=\bar{\lambda}$ \\
\hline
\end{tabular}

We will also need to recall the notion of isotropic vector. A nonzero vector $v$ in a scalar product space $\mathbb{K}^{n}$ is said to be isotropic if $\langle v, v\rangle_{\mathrm{M}}=0$, and nonisotropic if $\langle v, v\rangle_{\mathrm{M}} \neq 0$. Note that the set of all isotropic vectors in any scalar product space can be only one of the following three types: empty, all of $\mathbb{K}^{n} \backslash\{0\}$, or a nontrivial algebraic variety in $\mathbb{K}^{n} \backslash\{0\}$ (and hence a set of measure zero in $\mathbb{K}^{n}$ ). Then we have the following result.

Theorem 7.8. Suppose $A \in \mathbb{S}$, where $\mathbb{S}$ is any one of $\mathbb{G}, \mathbb{L}$, or $\mathbb{J}$ for a scalar product space $\mathbb{K}^{n}$. Let $x, y \in \mathbb{K}^{n}$ be eigenvectors for $A$ with $A x=\lambda x$ and $A y=\alpha y$. Then

(a) $\lambda \neq \wp(\alpha) \Rightarrow\langle x, y\rangle_{\mathrm{M}}=0$ ( $x$ and $y$ are " $M$-orthogonal"),

(b) $\lambda \neq \wp(\lambda) \Rightarrow x$ is an isotropic vector.

Proof. We give the proof only for $\mathbb{G}$ belonging to a sesquilinear form. The arguments for the other four cases are similar. We have

$$
\langle\lambda x, y\rangle_{\mathrm{M}}=\langle A x, y\rangle_{\mathrm{M}}=\left\langle x, A^{\star} y\right\rangle_{\mathrm{M}}=\left\langle x, A^{-1} y\right\rangle_{\mathrm{M}}=\langle x,(1 / \alpha) y\rangle_{\mathrm{M}} .
$$

Thus $\left(\bar{\lambda}-\frac{1}{\alpha}\right)\langle x, y\rangle_{\mathrm{M}}=0$ or, equivalently, $(\lambda-\wp(\alpha)) \overline{\langle x, y\rangle}_{\mathrm{M}}=0$ from the table above. Part (a) now follows immediately, and letting $x=y$ gives part (b).

Thus eigenvectors of structured matrices can be nonisotropic only if their eigenvalues are on the fixed point set of the corresponding pairing function. For sesquilinear forms these fixed point sets are the unit circle for $\mathbb{G}$, the imaginary axis for $\mathbb{L}$, and the real axis for $\mathbb{J}$. When a scalar product space contains no isotropic vectors at all, eigenvalues of structured matrices are all forced to be on these fixed point sets, constraining the eigenvalues even more than is generally the case. The simplest and best known example of this is the standard inner product $\langle x, y\rangle=x^{*} y$ on $\mathbb{C}^{n}$, where $\mathbb{G}$ is the unitary group with all eigenvalues on the unit circle, $\mathbb{L}$ is the skew-Hermitian matrices with all imaginary eigenvalues, and $\mathbb{J}$ is the Hermitian matrices with all real eigenvalues.

8. Structured singular value decomposition. The SVD is one of the most important factorizations in all of linear algebra. The SVD factors a matrix $A \in \mathbb{K}^{n \times n}$ into $A=U \Sigma V^{*}$, where $U, \Sigma, V \in \mathbb{K}^{n \times n}, U$ and $V$ are unitary, and $\Sigma$ is a diagonal matrix with only nonnegative entries [19], [25]. The factors $U, \Sigma$, and $V$ are never unique, although uniqueness is often forced on $\Sigma$ by adopting the convention that its diagonal entries are in nonincreasing order. For the structured matrices considered in this paper it is sometimes useful to relax this ordering convention or replace it by a different ordering convention.

In this section we will first discuss the extent to which the singular values of matrices in $\mathbb{G}, \mathbb{L}$, and $\mathbb{J}$ are structured and then survey what is known about structured SVDs.

8.1. Singular values of automorphisms. The singular values of matrices in many (but not all) automorphism groups have a pairing structure analogous to the eigenvalue pairings described in Theorem 7.2. 
THEOREM 8.1. Suppose $\mathbb{G}$ is the automorphism group of a scalar product and $A \in \mathbb{G}$ has a structured polar decomposition. Then $A$ has reciprocally paired singular values; i.e., the singular values of $A$ come in pairs, $\sigma$ and $1 / \sigma$, with the same multiplicity.

Proof. The singular values of $A$ are the same as the eigenvalues of the Hermitian factor $H$ in the structured polar decomposition $A=U H$. Since $H \in \mathbb{G}$, by Theorem 7.2 the eigenvalues of $H$, which are real and positive, have the reciprocal pairing property.

The converse of Theorem 8.1 does not hold. There are examples of automorphism groups $\mathbb{G}$ for which every $A \in \mathbb{G}$ has reciprocally paired singular values, but not every $A \in \mathbb{G}$ has a structured polar decomposition.

Example 8.2. Consider the automorphism group $\mathbb{G}$ of the bilinear form on $\mathbb{R}^{2}$ defined by $M=\left[\begin{array}{ll}1 & 0 \\ 0 & 4\end{array}\right]$. Since $\operatorname{det} A= \pm 1$ for every $A \in \mathbb{G}$ by Proposition 7.1, this forces every $A \in \mathbb{G}$ to have reciprocally paired singular values. However, one can easily verify that $A=\left[\begin{array}{ll}0 & 2 \\ \frac{1}{2} & 0\end{array}\right]$ is in $\mathbb{G}$ while $A^{*}$ is not, thus showing (by Theorem 5.7 ) that $A$ does not have a structured polar decomposition. More generally, for any scalar product on $\mathbb{R}^{n}$ defined by $M=\left[\begin{array}{cc}I_{n-1} & 0 \\ 0 & k\end{array}\right]$ with $k>0$, it can be shown that every $A \in \mathbb{G}$ has reciprocally paired singular values, but when $k \neq 1$ almost every $A \in \mathbb{G}$ fails to have a structured polar decomposition.

As an immediate consequence of Theorem 8.1 together with the results of section 5, we have the following large classes of automorphism groups in which every matrix has reciprocally paired singular values.

COROLlaRY 8.3. Let $\mathbb{G}$ be the automorphism group of a unitary scalar product, or of a scalar product $\langle\cdot, \cdot\rangle_{\mathrm{M}}$ with $M^{2}=\alpha I$ such that $M$ is real when $\langle\cdot, \cdot\rangle_{\mathrm{M}}$ is complex bilinear. Then every $A \in \mathbb{G}$ has reciprocally paired singular values.

Note that this result has previously been known for some particular groups, using different arguments in each case. For real pseudo-orthogonal matrices, see [22]; for symplectic matrices, see [14], [39], [49].

For scalar products that are neither unitary nor have $M^{2}=\alpha I$, the conclusion of Corollary 8.3 may or may not hold. For example, MATLAB experiments show that reciprocal pairing of singular values is absent when $M=\operatorname{diag}(1,2,3)$. With $M=\operatorname{diag}(1,1,2)$, though, numerical results indicate that singular value pairing is present-indeed, this pairing is a special case of Example 8.2. Characterizing the set of all scalar products whose automorphism groups have reciprocally paired singular values remains an open question.

Although it is not yet known precisely which automorphism groups have structured polar factors, or which automorphism groups have reciprocally paired singular values, Theorem 8.1 provides a necessary condition for the existence of a structured polar decomposition: Any automorphism that does not have the singular value reciprocal pairing property cannot have structured polar factors.

8.2. Singular values of matrices in $\mathbb{L}$ or $\mathbb{J}$. In general there is much less structure in the singular values of matrices in $\mathbb{L}$ or $\mathbb{J}$ than there is for automorphisms; real symmetric matrices and real Hamiltonian matrices are examples of Jordan and Lie algebras whose elements can have arbitrary singular values. On the other hand, there are also Lie and Jordan algebras in which all nonzero singular values of all matrices have even multiplicity. ${ }^{3}$ Both of these phenomena are explained by the

\footnotetext{
${ }^{3}$ Note that all of the examples of $\mathbb{L}$ and $\mathbb{J}$ in Table 2.1 exhibit one of these two behaviors, either no singular value structure at all or even multiplicity of all (nonzero) singular values.
} 
following result.

THEOREM 8.4. Let $\mathbb{S}$ be either the Lie algebra $\mathbb{L}$ or the Jordan algebra $\mathbb{J}$ of a scalar product $\langle\cdot, \cdot\rangle_{\mathrm{M}}$ on $\mathbb{K}^{n}$ that is both orthosymmetric and unitary. Suppose $A \in \mathbb{S}$, so that $A^{\star}=\kappa A$ where $\kappa= \pm 1$.

(a) For bilinear forms, $M^{T}=\varepsilon M$ where $\varepsilon= \pm 1$. If $\varepsilon \neq \kappa$, then the (nonzero) singular values of $A$ all have even multiplicity, while if $\varepsilon=\kappa$, then the singular values of matrices in $\mathbb{S}$ are unstructured; i.e., for an arbitrary list of $n$ nonnegative real numbers (repetitions allowed) there is some $A \in \mathbb{S}$ with these singular values.

(b) For sesquilinear forms, the singular values of matrices in either $\mathbb{L}$ or $\mathbb{J}$ are unstructured, in the same sense used in part (a).

Proof. (a) We begin by demonstrating the result for $M=I$, i.e., for

$$
\operatorname{Skew}(\mathbb{K}):=\left\{A \in \mathbb{K}^{n \times n}: A^{T}=-A\right\} \text { and } \operatorname{Sym}(\mathbb{K})=\left\{A \in \mathbb{K}^{n \times n}: A^{T}=A\right\} \text {. }
$$

For skew-symmetric $A, A^{*} A=-\bar{A} A$ is the product of two skew-symmetric matrices, and thus all its nonzero eigenvalues have even multiplicity [13]. Hence the nonzero singular values of $A$ all have even multiplicity. By contrast, the set $\operatorname{Sym}(\mathbb{K})$ contains the real symmetric positive semidefinite matrices, which clearly may have arbitrary singular values.

Next we invoke the orthosymmetry of $\langle\cdot, \cdot\rangle_{\mathrm{M}}$ to see that for any $A \in \mathbb{S}, M A$ is either symmetric or skew-symmetric. First, recall from property $(\mathrm{g})$ in Definition 2.4 that for any bilinear orthosymmetric scalar product $\langle\cdot, \cdot\rangle_{\mathrm{M}}, M^{T}=\varepsilon M$ where $\varepsilon= \pm 1$. Then $A \in \mathbb{S} \Rightarrow \kappa A=A^{\star}=M^{-1} A^{T} M \Rightarrow M A=\kappa A^{T} M$, and thus $(M A)^{T}=$ $\kappa\left(A^{T} M\right)^{T}=\varepsilon \kappa(M A)$. By Definition 2.4 (f) we know that $\mathbb{K}^{n \times n}=\mathbb{L} \oplus \mathbb{J}$, and hence left multiplication by $M$ is a bijection from $\mathbb{K}^{n \times n}$ to $\mathbb{K}^{n \times n}$ that maps $\mathbb{L}$ and $\mathbb{J}$ to $\operatorname{Skew}(\mathbb{K})$ and $\operatorname{Sym}(\mathbb{K})$. To be more precise, we have shown that

$$
M \cdot \mathbb{S}= \begin{cases}\operatorname{Skew}(\mathbb{K}) & \text { if } \varepsilon \neq \kappa, \\ \operatorname{Sym}(\mathbb{K}) & \text { if } \varepsilon=\kappa\end{cases}
$$

or, equivalently,

$$
\mathbb{S}= \begin{cases}M^{-1} \cdot \operatorname{Skew}(\mathbb{K}) & \text { if } \varepsilon \neq \kappa \\ M^{-1} \cdot \operatorname{Sym}(\mathbb{K}) & \text { if } \varepsilon=\kappa .\end{cases}
$$

Finally, $\langle\cdot, \cdot\rangle_{\mathrm{M}}$ being a unitary scalar product means that $M^{-1}=\alpha U$ for some unitary $U$ and $\alpha>0$, by Definition 2.3, property (d). From (8.2) we can therefore conclude that the singular value structure of $\mathbb{S}$ is the same as that of $\operatorname{Skew}(\mathbb{K})$ or $\operatorname{Sym}(\mathbb{K})$, depending only on whether $\varepsilon$ and $\kappa$ are equal or not.

(b) For sesquilinear forms, orthosymmetry of $\langle\cdot, \cdot\rangle_{\mathrm{M}}$ means that $M^{*}=\gamma M$ for some $|\gamma|=1$ (see Definition 2.4(g)). Choosing $\beta$ so that $\beta^{2}=\gamma$, an argument similar to the one in part (a) shows that for any $A \in \mathbb{S}, \beta M A$ is either Hermitian or skewHermitian. But the set of all skew-Hermitian matrices is $i \cdot \operatorname{Herm}(\mathbb{C})$, where $\operatorname{Herm}(\mathbb{C})$ denotes the set of Hermitian matrices. Combining this with $\mathbb{C}^{n \times n}=\mathbb{L} \oplus \mathbb{J}$, we have the following analogue of (8.2):

$$
\mathbb{S}= \begin{cases}i \bar{\beta} M^{-1} \cdot \operatorname{Herm}(\mathbb{C}) & \text { if } \kappa=-1 \\ \bar{\beta} M^{-1} \cdot \operatorname{Herm}(\mathbb{C}) & \text { if } \kappa=+1\end{cases}
$$

Since $\langle\cdot, \cdot\rangle_{\mathrm{M}}$ is also unitary we have $M^{-1}=\alpha U$ for some unitary $U$ and $\alpha>0$. Thus in all cases we have $\mathbb{S}=\widetilde{U} \cdot \operatorname{Herm}(\mathbb{C})$ for some unitary $\widetilde{U}$, so the singular 
value structure of every $\mathbb{S}$ is the same as that of $\operatorname{Herm}(\mathbb{C})$. But the singular values of matrices in Herm $(\mathbb{C})$ are unstructured, since Herm $(\mathbb{C})$ contains all real symmetric positive semidefinite matrices, which may have arbitrary singular values.

8.3. Structured SVD of automorphisms. In this section we survey (without proof) some recent results on the structured SVD question:

For which scalar products does every matrix in the corresponding automorphism group $\mathbb{G}$ have a "structured" SVD, that is, an SVD $U \Sigma V^{*}$ such that $U, \Sigma, V \in \mathbb{G}$ ?

By contrast with the other structured factorizations considered in this paper, there is as yet no unified treatment of the structured SVD question for any large class of scalar products. However, for particular scalar products there have recently been some positive results, using arguments specific to the situation in each case. The most important of these results are for the three varieties of symplectic matrices seen in Table 2.1.

THEOREM 8.5 (symplectic SVD). Let $\mathbb{S}$ be either the real symplectic, complex symplectic, or conjugate symplectic group of $2 n \times 2 n$ matrices. Then every $A \in \mathbb{S}$ has an $S V D, A=U \Sigma V^{*}$, where the factors $U, \Sigma$, and $V$ are also in $\mathbb{S}$. Furthermore, $\Sigma$ can always be chosen to be of the form $\Sigma=\operatorname{diag}\left(D, D^{-1}\right)$, where $D$ is an $n \times n$ diagonal matrix with $d_{11} \geq d_{22} \geq \cdots \geq d_{n n} \geq 1$. This $\Sigma$ is unique.

This theorem can be found in [49] for real symplectic and conjugate symplectic matrices, in [14] for complex symplectic matrices, and with a different proof in [39] for all three types of symplectic matrices. Structure-preserving Jacobi algorithms for computing a symplectic SVD of a real or complex symplectic matrix are developed in [10]. Theorem 8.5 also has an analytic version, as shown in [39].

TheOREM 8.6 (analytic symplectic SVD). Let $\mathbb{S}$ be as in Theorem 8.5, and suppose $A(t) \in \mathbb{S}$ for $t \in[a, b]$ is an analytically varying symplectic matrix. Then there is an analytic $S V D, A(t)=U(t) \Sigma(t) V(t)^{*}$, such that $U(t), \Sigma(t), V(t) \in \mathbb{S}$ for all $t \in[a, b]$.

One class of automorphism groups $\mathbb{G}$ for which completely structured SVDs are not possible are the pseudo-orthogonal groups, also known as the Lorentz groups. The only positive diagonal matrix in these groups is the identity matrix; thus no nonorthogonal Lorentz matrix can ever have an SVD in which all three factors are in $\mathbb{G}$. However, there is a closely related structured decomposition of Lorentz matrices from which the singular values and vectors can be easily read off.

TheOREM 8.7 (hyperbolic CS decomposition). Let $\mathbb{G}$ be one of the pseudoorthogonal groups $O(p, q, \mathbb{R})$; here without loss of generality we will assume that $p \leq q$. Then any $A \in \mathbb{G}$ has a "hyperbolic $C S$ decomposition"; that is, A has a factorization $A=U \widetilde{\Sigma} V^{T}$, where $U, V, \widetilde{\Sigma} \in \mathbb{G}, U$ and $V$ are orthogonal, and $\widetilde{\Sigma}$ has the form

$$
\widetilde{\Sigma}=\left[\begin{array}{rrc}
C & -S & 0 \\
-S & C & 0 \\
0 & 0 & I_{q-p}
\end{array}\right]
$$

with $p \times p$ diagonal blocks $C$ and $S$ such that $C^{2}-S^{2}=I$ and $c_{i i}>s_{i i} \geq 0$ for $i=1, \ldots, p$.

Theorem 8.7 can be found in [22], together with references to earlier work on this factorization. An equivalent result is proved in [11] and also extended to the case of a smoothly varying Lorentz matrix $A(t)$, albeit with the restriction that the numbers $s_{i i}(t)$ remain distinct for all $t$. Note that this restriction is equivalent to assuming that 
the singular values of the block matrix $\left[\begin{array}{cc}C & -S \\ -S & C\end{array}\right]$ remain distinct for all $t$. Theorem 8.7 can also be easily extended to the pseudo-unitary groups $U(p, q, \mathbb{C})$.

Characterizing which automorphism groups $\mathbb{G}$ have structured SVDs and which do not, as well as which $\mathbb{G}$ have decompositions that are "almost" structured SVDs, e.g., structured "CS-like" decompositions, is still an open question.

8.4. Structured SVD-like decomposition in Lie and Jordan algebras. Since $\mathbb{L}$ and $\mathbb{J}$ are not closed under multiplication, we cannot in general expect SVDs of such matrices to have all three factors in $\mathbb{L}$ or $\mathbb{J}$. Instead we aim to find SVDlike decompositions that reflect the $\mathbb{L} / \mathbb{J}$ structure of the given matrix in a manner analogous to the symmetric SVD of complex symmetric matrices [9], also known as the Takagi factorization [25]. Let us begin by recalling this factorization and its skew-symmetric analogue. Note that $\operatorname{Sym}(\mathbb{K})$ and $\operatorname{Skew}(\mathbb{K})$ are as defined in (8.1).

TheOREM 8.8 (symmetric SVD/Takagi factorization). Let $A \in \operatorname{Sym}(\mathbb{C})$. Then there exist a real nonnegative diagonal matrix $\Sigma=\operatorname{diag}\left(\sigma_{1}, \ldots, \sigma_{n}\right)$ and a unitary matrix $U$ such that $A=U \Sigma U^{T}$.

We refer to [25, Cor. 4.4.4] for a proof and to [25, p. 218] and [26, sect. 3.0] for references to earlier work on this factorization. Note that the Takagi factorization is a special SVD $A=U \Sigma V^{*}$ in which $V=\bar{U}$. The $\sigma_{i}$ are the singular values of $A$, and the columns of $U=\left[u_{1}, \ldots, u_{n}\right]$ are the corresponding left singular vectors; that is, $A \bar{u}_{i}=\sigma_{i} u_{i}$.

Theorem 8.9 (skew-symmetric Takagi factorization). Suppose $A \in \operatorname{Skew}(\mathbb{C})$. Then there exist a unitary matrix $U$ and a block diagonal matrix

$$
\Sigma=D_{1} \oplus \cdots \oplus D_{k} \oplus 0 \oplus \cdots \oplus 0
$$

such that $A=U \Sigma U^{T}$. Here $D_{j}=\left[\begin{array}{cc}0 & z_{j} \\ -z_{j} & 0\end{array}\right]$ with nonzero $z_{j} \in \mathbb{C}$ for $j=1, \ldots, k$. If $A \in \operatorname{Skew}(\mathbb{R})$, then $U$ can be chosen to be real orthogonal and the $z_{j}$ to be real. Note that the nonzero singular values of $A$ are $\left|z_{j}\right|$, each with multiplicity two.

For a proof of this result see [25, sect. 4.4, problems 25-26].

Although the skew-symmetric Takagi factorization is not literally an SVD, it is an example of something that might reasonably be termed an "SVD-like" decomposition, since the $\Sigma$-factor reveals the singular values of $A$ in a simple and straightforward fashion. Similarly, the real case of Theorem 8.8 may be viewed as an SVD-like decomposition. Any real symmetric $A$ may be factored as $A=U \Sigma U^{T}$ with $U$ real orthogonal and $\Sigma$ real diagonal, but in general one cannot arrange for all the entries of $\Sigma$ to be nonnegative. Nevertheless the singular values of $A$ are easily recovered from $\Sigma$ by taking absolute values. It is in this sense that we seek SVD-like decompositions for matrices in $\mathbb{L}$ and $\mathbb{J}$.

We also want a factorization that reflects the structure of the given matrix from $\mathbb{L}$ or $\mathbb{J}$. Observe that the symmetric/skew-symmetric structure of the matrix $A$ in the Takagi factorizations is not expressed by structure in each individual factor, but rather by the overall form of the decomposition, i.e., as a congruence of a condensed symmetric or skew-symmetric matrix. In a scalar product space the analogue of congruence is $\star$-congruence, and it is not hard to see that $\mathbb{L}$ and $\mathbb{J}$-structure is preserved by arbitrary $\star$-congruence whenever the scalar product is orthosymmetric; that is, for $\mathbb{S}=\mathbb{L}$ or $\mathbb{J}$ and any $P \in \mathbb{K}^{n \times n}, A \in \mathbb{S} \Rightarrow P A P^{\star} \in \mathbb{S}$. Indeed, this property gives another characterization of orthosymmetric scalar products (see Theorem A.5(f)). The next result gives a "Takagi-like" factorization for any $A \in \mathbb{L}$ or $\mathbb{J}$ using $\star$-congruence of matrices in $\mathbb{L}$ or $\mathbb{J}$ that are simple enough to reveal the singular values of $A$. Note that we consider only scalar products that are both orthosymmetric and unitary. 
TheOREM 8.10 (structured SVD-like decomposition for $\mathbb{L}$ and $\mathbb{J})$. Let $\mathbb{S}$ be either the Lie algebra $\mathbb{L}$ or Jordan algebra $\mathbb{J}$ of a scalar product $\langle\cdot, \cdot\rangle_{\mathrm{M}}$ on $\mathbb{K}^{n}$ that is both orthosymmetric and unitary. Then every $A \in \mathbb{S}$ has an $S V D$-like decomposition of the form $A=U \Sigma U^{\star}$, where $U$ is unitary and $\Sigma \in \mathbb{S}$ reveals the singular values of $A$ in the following sense: with $\alpha>0$ such that $\alpha M$ is unitary, the matrix $\alpha M \Sigma$ has the same singular values as $A$ and is either diagonal or block diagonal with diagonal blocks of the form $\left[\begin{array}{cc}0 & z \\ -z & 0\end{array}\right]$. If $\mathbb{K}=\mathbb{R}$, then $U$ and $\Sigma$ may be chosen to be real.

Proof. Orthosymmetry of a bilinear $\langle\cdot, \cdot\rangle_{\mathrm{M}}$ means that $M^{T}=\varepsilon M$ with $\varepsilon= \pm 1$. Also by definition $A \in \mathbb{S} \Rightarrow A^{\star}=\kappa A$ with $\kappa= \pm 1$. Thus if $\widetilde{A}:=M A$, then by (8.2) we have $\widetilde{A} \in \widetilde{\mathbb{S}}$, where

$$
\widetilde{\mathbb{S}}= \begin{cases}\operatorname{Skew}(\mathbb{K}) & \text { if } \varepsilon \neq \kappa \\ \operatorname{Sym}(\mathbb{K}) & \text { if } \varepsilon=\kappa\end{cases}
$$

From Theorems 8.8 and 8.9 there exist $V$ unitary and $\widetilde{\Sigma} \in \widetilde{\mathbb{S}}$ such that $\widetilde{A}=V \widetilde{\Sigma} V^{T}$; this $\widetilde{\Sigma}$ is real diagonal when $\widetilde{\mathbb{S}}=\operatorname{Sym}(\mathbb{K})$, and block diagonal as in (8.4) when $\widetilde{\mathbb{S}}=$ $\operatorname{Skew}(\mathbb{K})$. In either case, $\widetilde{\Sigma}$ reveals the singular values of $\widetilde{A}$. From this factorization of $\widetilde{A}$ we get

$$
A=M^{-1} \widetilde{A}=M^{-1} V \widetilde{\Sigma} V^{T}=\left(M^{-1} V M\right) M^{-1} \widetilde{\Sigma} V^{T}=\left(V^{T}\right)^{\star}\left(M^{-1} \widetilde{\Sigma}\right) V^{T}=W^{\star} \Sigma W,
$$

where $W=V^{T}$ is unitary and $\Sigma=M^{-1} \widetilde{\Sigma} \in \mathbb{S}$.

A similar argument also leads to a factorization $A=W^{\star} \Sigma W$ when $\langle\cdot, \cdot\rangle_{\mathrm{M}}$ is sesquilinear. In this case, orthosymmetry means that $M^{*}=\gamma M$ for some $|\gamma|=1$. Using once again that $A \in \mathbb{S} \Rightarrow A^{\star}=\kappa A$ with $\kappa= \pm 1$, we have by (8.3) that $\widetilde{A}:=\sqrt{\gamma} M A$ is an element of

$$
\widetilde{\mathbb{S}}= \begin{cases}\operatorname{Herm}(\mathbb{C}) & \text { if } \kappa=1 \\ i \cdot \operatorname{Herm}(\mathbb{C}) & \text { if } \kappa=-1\end{cases}
$$

Hence there exist a unitary $V$ and diagonal $\widetilde{\Sigma} \in \widetilde{\mathbb{S}}$ such that $\widetilde{A}=V \widetilde{\Sigma} V^{*}$. Thus

$$
A=\gamma^{-1 / 2} M^{-1} \widetilde{A}=\gamma^{-1 / 2}\left(M^{-1} V M\right) M^{-1} \widetilde{\Sigma} V^{*}=\left(V^{*}\right)^{\star}\left(\gamma^{-1 / 2} M^{-1} \widetilde{\Sigma}\right) V^{*}=W^{\star} \Sigma W,
$$

where $W=V^{*}$ is unitary and $\Sigma=\gamma^{-1 / 2} M^{-1} \widetilde{\Sigma} \in \mathbb{S}$.

In both the bilinear and sesquilinear cases, the Takagi-like form $A=U \Sigma U^{\star}$ with $U$ unitary is now obtained by letting $U=W^{\star}$ and using the involutory property (e) in Definition 2.4 for orthosymmetric scalar products.

Finally, we invoke the hypothesis that $\langle\cdot, \cdot\rangle_{\mathrm{M}}$ is unitary to see how the factor $\Sigma$ reveals the singular values of $A=U \Sigma U^{\star}$. Since $U$ is unitary, so is $U^{\star}$ by Definition 2.3(b), and thus $A$ and $\Sigma$ share the same singular values. Property (d) in Definition 2.3 gives us an $\alpha>0$ such that $\alpha M$ is unitary, and thus $\alpha M \Sigma$ also has the same singular values as $A$. But $\alpha M \Sigma$, being a scalar multiple of $\widetilde{\Sigma}$, is either diagonal or block diagonal of the form (8.4), and thus reveals the singular values of $A$.

9. Concluding remarks. This work adds to the growing literature on matrices in automorphism groups, Lie algebras, and Jordan algebras associated with a scalar product. Results on structured factorizations have been given here with as few assumptions on the scalar products as possible. One of our main contributions is the recognition that for many results the appropriate assumption is that the scalar 
product is either unitary or orthosymmetric, and we have given a number of different characterizations of these two classes of scalar products.

Some open questions have been identified. A major area for future work concerns the numerical computation of structured factorizations in a way that exploits - and retains, in the presence of rounding errors - the structure. Some recent work along these lines can be found in the references, but much remains to be done.

\section{Appendix A. Special classes of scalar products.}

Many useful properties, such as the involutory property $\left(A^{\star}\right)^{\star}=A$, do not hold in every scalar product space. In this appendix we consider a number of such properties, including all those listed earlier in section 2.3, and show that they cluster together into two groups of equivalent properties, thereby delineating two natural classes of scalar products. We have termed these classes orthosymmetric and unitary, and believe that their identification has simplified the presentation in this paper and will also help to clarify existing results in the literature. Note that all the "classical" examples of scalar products listed in Table 2.1 are both orthosymmetric and unitary, as will easily be seen from Theorems A.5 and A.7.

A.1. Preliminaries. To demonstrate the equivalence of various scalar product properties, we need a flexible way to detect when a matrix $A$ is a scalar multiple of the identity. It is well known that when $A$ commutes with all of $\mathbb{K}^{n \times n}$, then $A=\alpha I$ for some $\alpha \in \mathbb{K}$. There are many other sets besides $\mathbb{K}^{n \times n}$, though, that suffice to give the same conclusion.

Definition A.1. A set of matrices $\mathcal{S} \subseteq \mathbb{K}^{n \times n}$ will be called a CS-set for $\mathbb{K}^{n \times n}$ if the centralizer of $\mathcal{S}$ consists only of the scalar multiples ${ }^{4}$ of $I$; that is,

$$
B S=S B \text { for all } S \in \mathcal{S} \Longrightarrow B=\alpha I \text { for some } \alpha \in \mathbb{K} .
$$

The following lemma describes a number of useful examples ${ }^{5}$ of CS-sets for $\mathbb{R}^{n \times n}$ and $\mathbb{C}^{n \times n}$. For this lemma, $D$ denotes an arbitrary diagonal matrix in $\mathbb{K}^{n \times n}$ with distinct diagonal entries, $D_{+}$denotes a diagonal matrix with distinct positive diagonal entries,

$$
N=\left[\begin{array}{lllll}
0 & 1 & & \\
& 0 & 1 & \\
& & \ddots & \\
& & & 1 \\
& & & 0
\end{array}\right]
$$

is the $n \times n$ nilpotent Jordan block,

$$
C=\left[\begin{array}{lllll}
0 & 1 & & & \\
& 0 & 1 & \\
& & \cdots & \\
& & & 1 \\
1 & & & 0
\end{array}\right]
$$

is the cyclic permutation matrix, $E=\left[\begin{array}{ll}0 & 1 \\ 1 & 0\end{array}\right] \oplus I_{n-2}$, and $F=\left[\begin{array}{cc}0 & -1 \\ 1 & 0\end{array}\right] \oplus I_{n-2}$.

Lemma A.2. Suppose $\mathcal{S} \subseteq \mathbb{K}^{n \times n}$. Then the following hold.

(a) $\mathcal{S}$ contains a CS-set $\Rightarrow \mathcal{S}$ is a CS-set.

(b) Let $\overline{\mathcal{S}}$ denote $\{\bar{A}: A \in \mathcal{S}\}$. If $\mathcal{S}$ is a $C S$-set for $\mathbb{C}^{n \times n}$, then so is $\overline{\mathcal{S}}$.

\footnotetext{
${ }^{4}$ One may think of "CS" as standing for either "Commuting implies $\underline{S}$ calar," or "Centralizer equals the $\underline{S}$ calars."

${ }^{5}$ Another important source of CS-sets for $\mathbb{C}^{n \times n}$ is the classical "Schur's lemma" [34], [48] from representation theory: Any $\mathcal{S} \subseteq \mathbb{C}^{n \times n}$ for which there is no nontrivial $\mathcal{S}$-invariant subspace in $\mathbb{C}^{n}$ is a CS-set for $\mathbb{C}^{n \times n}$. Thus the matrices in any irreducible representation of a finite group will be a CS-set.
} 
(c) Any vector space basis for $\mathbb{K}^{n \times n}$ or algebra generating set for $\mathbb{K}^{n \times n}$ is a CSset for $\mathbb{K}^{n \times n}$. More generally, any set whose span (either in the vector space sense or the algebra sense) contains a CS-set is a CS-set.

(d) Each of the finite sets $\{D, N\},\left\{D, N+N^{T}\right\},\left\{D_{+}, 3 I+N+N^{T}\right\}$, and $\{C, E, F\}$ is a CS-set for $\mathbb{K}^{n \times n}$.

(e) Any open subset $\mathcal{S} \subseteq \mathbb{K}^{n \times n}$ is a CS-set. (Indeed any open subset of $\mathbb{R}^{n \times n}$ is a $C S$-set for $\mathbb{C}^{n \times n}$.)

(f) The sets of all unitary matrices, all Hermitian matrices, all Hermitian positive semidefinite matrices, and all Hermitian positive definite matrices are $C S$-sets for $\mathbb{C}^{n \times n}$. The sets of all real orthogonal matrices and all real symmetric matrices are CS-sets for $\mathbb{R}^{n \times n}$ and for $\mathbb{C}^{n \times n}$.

Proof.

(a) This is an immediate consequence of Definition A.1.

(b) $B \bar{S}=\bar{S} B$ for all $\bar{S} \in \overline{\mathcal{S}} \Rightarrow \bar{B} S=S \bar{B}$ for all $S \in \mathcal{S}$. But $\mathcal{S}$ is a CS-set, so $\bar{B}=\alpha I$ or, equivalently, $B=\bar{\alpha} I$. Thus $\overline{\mathcal{S}}$ is a CS-set.

(c) If $B$ commutes with either a vector space basis or an algebra generating set for $\mathbb{K}^{n \times n}$, then it commutes with all of $\mathbb{K}^{n \times n}$, and hence $B=\alpha I$.

(d) Any matrix $B$ that commutes with $D$ must itself be a diagonal matrix, and any diagonal $B$ that commutes with $N$ must have equal diagonal entries, so that $B=\alpha I$. Thus $\mathcal{S}=\{D, N\}$ is a CS-set. Similar arguments show that $\left\{D, N+N^{T}\right\}$ and $\left\{D_{+}, 3 I+N+N^{T}\right\}$ are also CS-sets. To see that $\{C, E, F\}$ is a CS-set, first observe that a matrix $B$ commutes with $C$ if and only if it is a polynomial in $C$, i.e., if and only if $B$ is a circulant matrix. But any circulant $B$ that commutes with $E$ must be of the form $B=\alpha I+\beta K$, where $K$ is defined by

$$
K_{i j}= \begin{cases}0 & \text { if } i=j \\ 1 & \text { if } i \neq j\end{cases}
$$

Finally, $B=\alpha I+\beta K$ commuting with $F$ forces $\beta=0$, and thus $B=\alpha I$, showing that $\{C, E, F\}$ is a CS-set.

(e) This follows from (a) and (c), since any open subset of $\mathbb{K}^{n \times n}$ contains a vector space basis for $\mathbb{K}^{n \times n}$.

(f) This follows from (a) and (d) by observing that $\left\{D, N+N^{T}\right\}$ consists of two real symmetric matrices, $\left\{D_{+}, 3 I+N+N^{T}\right\}$ consists of two real symmetric positive definite matrices, and $\{C, E, F\}$ consists of three real orthogonal matrices.

A second simple result that is needed to show the equivalence of various scalar product properties is the following lemma.

LemmA A.3. Let $M \in \mathbb{K}^{n \times n}$ be a nonzero matrix. Then

1. $M^{T}=\alpha M$ for some $\alpha \in \mathbb{K} \Leftrightarrow M^{T}= \pm M$,

2. $M^{*}=\alpha M$ for some $\alpha \in \mathbb{K} \Leftrightarrow M^{*}=\alpha M$ for some $|\alpha|=1$

$$
\Leftrightarrow M=\beta H \text { for some Hermitian } H \text { and }|\beta|=1 \text {, }
$$

3. $M M^{*}=\alpha I$ for some $\alpha \in \mathbb{K} \Leftrightarrow M=\beta U$ for some unitary $U$ and $\beta>0$.

Proof. Since the proofs of the reverse implications $(\Leftarrow)$ in 1,2 , and 3 are immediate, we include only the proofs of the forward implications $(\Rightarrow)$ in each case.

1. $M^{T}=\alpha M \Rightarrow M=\left(M^{T}\right)^{T}=(\alpha M)^{T}=\alpha M^{T}=\alpha^{2} M \Rightarrow \alpha^{2}=1 \Rightarrow \alpha= \pm 1$.

2. $M^{*}=\alpha M \Rightarrow M=\left(M^{*}\right)^{*}=(\alpha M)^{*}=\bar{\alpha} M^{*}=|\alpha|^{2} M \Rightarrow|\alpha|^{2}=1 \Rightarrow|\alpha|=1$. To see the second implication, let $H=\sqrt{\alpha} M$, where $\sqrt{\alpha}$ is either of the two square roots of $\alpha$ on the unit circle. It is easy to check that $H$ is Hermitian, and that $M=\beta H$ with $\beta=(\sqrt{\alpha})^{-1}$ on the unit circle. 
3. $M M^{*}$ is positive semidefinite, so $\alpha \geq 0$; then $M \neq 0$ implies $\alpha>0$. It follows that $U=\frac{1}{\sqrt{\alpha}} M$ is unitary, and thus $M=\beta U$ with $\beta=\sqrt{\alpha}>0$.

A.2. Orthosymmetric scalar products. In Shaw [48], scalar products that enjoy property (b) in Theorem A.5 are called "orthosymmetric." We adopt this name in the following definition, which extends Definition 2.4.

DEFINITION A.4 (orthosymmetric scalar product). A scalar product is said to be orthosymmetric if it satisfies any one (and hence all) of the seven equivalent properties in Theorem A.5.

TheOREM A.5. For a scalar product $\langle\cdot, \cdot\rangle_{\mathrm{M}}$ on $\mathbb{K}^{n}$, the following are equivalent:

(a) Adjoint with respect to $\langle\cdot, \cdot\rangle_{\mathrm{M}}$ is involutory; that is, $\left(A^{\star}\right)^{\star}=A$ for all $A \in$ $\mathbb{K}^{n \times n}$.

(a') $\left(A^{\star}\right)^{\star}=A$ for all $A$ in some $C S$-set for $\mathbb{K}^{n \times n}$.

(b) Vector orthogonality is a symmetric relation; that is,

(c) $\mathbb{K}^{n \times n}=\mathbb{L} \oplus \mathbb{J}$.

$$
\langle x, y\rangle_{\mathrm{M}}=0 \Longleftrightarrow\langle y, x\rangle_{\mathrm{M}}=0 \text { for all } x, y \in \mathbb{K}^{n} .
$$

(d) For bilinear forms, $M^{T}= \pm M$. For sesquilinear forms, $M^{*}=\alpha M$ with $\alpha \in \mathbb{C},|\alpha|=1$; equivalently, $M=\beta H$ with $\beta \in \mathbb{C},|\beta|=1$, and Hermitian $H$.

(e) There exists some CS-set for $\mathbb{K}^{n \times n}$ with the property that every matrix $A$ in this $C S$-set can be factored as $A=W S$ with $W \in \mathbb{G}$ and $S \in \mathbb{J}$.

(f) $\mathbb{L}$ and $\mathbb{J}$ are preserved by arbitrary $\star$-congruence; that is, for $\mathbb{S}=\mathbb{L}$ or $\mathbb{J}$ and $P \in \mathbb{K}^{n \times n}, B \in \mathbb{S} \Rightarrow P B P^{\star} \in \mathbb{S}$.

Proof. Using (2.2) we have

$$
\left(A^{\star}\right)^{\star}= \begin{cases}\left(M^{-1} M^{T}\right) A\left(M^{-1} M^{T}\right)^{-1} & \text { for bilinear forms, } \\ \left(M^{-1} M^{*}\right) A\left(M^{-1} M^{*}\right)^{-1} & \text { for sesquilinear forms. }\end{cases}
$$

Hence,

$$
\left(A^{\star}\right)^{\star}=A \Longleftrightarrow \begin{cases}\left(M^{-1} M^{T}\right) A=A\left(M^{-1} M^{T}\right) & \text { for bilinear forms, } \\ \left(M^{-1} M^{*}\right) A=A\left(M^{-1} M^{*}\right) & \text { for sesquilinear forms. }\end{cases}
$$

$\underline{(\mathrm{a}) \Leftrightarrow\left(\mathrm{a}^{\prime}\right) \Leftrightarrow(\mathrm{d})}$

(a) $\Rightarrow\left(\mathrm{a}^{\prime}\right)$ : Obvious.

$\left(\mathrm{a}^{\prime}\right) \Rightarrow(\mathrm{d})$ : Equation (A.2) holding for all $A$ in some CS-set means that $M^{-1} M^{T}=$ $\alpha I$ (resp., $M^{-1} M^{*}=\alpha I$ ). The desired conclusion now follows from Lemma A.3.

(d) $\Rightarrow$ (a): This follows from a straightforward substitution into (A.1). (a) $\Leftrightarrow(\mathrm{c})$

(a) $\Rightarrow$ (c): For any scalar product, $\mathbb{L} \cap \mathbb{J}=\{0\} ;$ if $B \in \mathbb{L} \cap \mathbb{J}$, then $-B=B^{\star}=B$, and thus $B=0$. Now suppose that (a) holds and consider an arbitrary $A \in \mathbb{K}^{n \times n}$. Define $L=\frac{1}{2}\left(A-A^{\star}\right)$ and $S=\frac{1}{2}\left(A+A^{\star}\right)$ so that $A=L+S$. From $\left(A^{\star}\right)^{\star}=A$, we conclude that $L^{\star}=-L$ so that $L \in \mathbb{L}$. Similarly one sees that $S \in \mathbb{J}$. The decomposition $A=L+S$ shows that $\mathbb{K}^{n \times n}=\mathbb{L}+\mathbb{J}$ and because $\mathbb{L} \cap \mathbb{J}=\{0\}$, the sum is direct.

(c) $\Rightarrow$ (a): $A=L+S \Rightarrow A^{\star}=L^{\star}+S^{\star}=-L+S \Rightarrow\left(A^{\star}\right)^{\star}=(-L)^{\star}+S^{\star}=$ $L+S=A$.

(b) $\Leftrightarrow(\mathrm{d})$

(b) $\Rightarrow(\mathrm{d})$ : Suppose $\langle\cdot, \cdot\rangle_{\mathrm{M}}$ is a bilinear form. Letting $y=M w$, we have

$$
x^{T} y=0 \Leftrightarrow x^{T} M w=0 \stackrel{(\mathrm{b})}{\Longleftrightarrow} w^{T} M x=0 \Leftrightarrow x^{T} M^{T} w=0 \Leftrightarrow x^{T}\left(M^{T} M^{-1}\right) y=0 .
$$


A similar argument for sesquilinear forms shows that $x^{*} y=0 \Leftrightarrow x^{*}\left(M^{*} M^{-1}\right) y=0$. Thus, property (b) implies that

$$
\langle x, y\rangle_{\mathrm{I}}=0 \Leftrightarrow\langle x, y\rangle_{\mathrm{B}}=0, \text { where } B= \begin{cases}M^{T} M^{-1} & \text { for bilinear forms, } \\ M^{*} M^{-1} & \text { for sesquilinear forms. }\end{cases}
$$

Using this relationship we can now probe the entries of $B$ with various pairs $x, y$ such that $\langle x, y\rangle_{\mathrm{I}}=0$. Let $x=e_{i}$ and $y=e_{j}$ with $i \neq j$. Then $B_{i j}=\left\langle e_{i}, e_{j}\right\rangle_{\mathrm{B}}=0$, and thus $B$ must be a diagonal matrix. Next, let $x=e_{i}+e_{j}$ and $y=e_{i}-e_{j}$ with $i \neq j$. Then

$$
0=\left\langle e_{i}+e_{j}, e_{i}-e_{j}\right\rangle_{\mathrm{B}}=B_{i i}+B_{j i}-B_{i j}-B_{j j}=B_{i i}-B_{j j} ;
$$

hence $B_{i i}=B_{j j}$ for all $i \neq j$. Thus $B=\alpha I$ for some nonzero $\alpha \in \mathbb{K}$, and the desired conclusion follows from Lemma A.3.

$(d) \Rightarrow(b)$ : This direction is a straightforward verification. For bilinear forms,

$$
\langle x, y\rangle_{\mathrm{M}}=0 \Leftrightarrow x^{T} M y=0 \Leftrightarrow\left(x^{T} M y\right)^{T}=0 \Leftrightarrow \pm\left(y^{T} M x\right)=0 \Leftrightarrow\langle y, x\rangle_{\mathrm{M}}=0,
$$

and for sesquilinear forms,

$$
\langle x, y\rangle_{\mathrm{M}}=0 \Leftrightarrow x^{*} M y=0 \Leftrightarrow\left(x^{*} M y\right)^{*}=0 \Leftrightarrow \alpha\left(y^{*} M x\right)=0 \Leftrightarrow\langle y, x\rangle_{\mathrm{M}}=0 .
$$

$\underline{(\mathrm{e}) \Leftrightarrow(\mathrm{a})}$

(e) $\Rightarrow$ (a): The proof of this equivalence is just a slightly modified version of the proof of Theorem 6.4. For all $A$ in our CS-set we have

$$
\left(A^{\star}\right)^{\star}=\left(S^{\star} W^{\star}\right)^{\star}=\left(S W^{-1}\right)^{\star}=W^{-\star} S^{\star}=\left(W^{-1}\right)^{-1} S=W S=A,
$$

and thus (a') holds. That (a') implies (a) was shown earlier.

(a) $\Rightarrow$ (e): The continuity of the eigenvalues of $A^{\star} A$ implies that there is an open neighborhood $\mathcal{U}$ of the identity in which $A^{\star} A$ has no eigenvalues on $\mathbb{R}^{-}$. Thus by Theorem 6.2 every $A$ in the CS-set $\mathcal{U}$ can be factored as $A=W S$ with $W \in \mathbb{G}$ and $S \in \mathbb{J}$.

(a) $\Leftrightarrow(\mathrm{f})$

$(\mathrm{a}) \Rightarrow(\mathrm{f})$ : Let $B \in \mathbb{S}$ so that $B^{\star}= \pm B$. Then $\left(P B P^{\star}\right)^{\star}=\left(P^{\star}\right)^{\star} B^{\star} P^{\star}=$ $\pm P B P^{\star}$, and thus $P B P^{\star} \in \mathbb{S}$.

(f) $\Rightarrow$ (a): Consider $\mathbb{S}=\mathbb{J}$ and $B=I \in \mathbb{J}$. Then (f) implies that $P P^{\star} \in \mathbb{J}$ for any $P \in \mathbb{K}^{n \times n}$, and thus $P P^{\star}=\left(P P^{\star}\right)^{\star}=\left(P^{\star}\right)^{\star} P^{\star}$. Since $P^{\star}$ is nonsingular for any nonsingular $P$, we have $P=\left(P^{\star}\right)^{\star}$ for every nonsingular $P$. Thus by Lemma A.2(e) we have property $\left(\mathrm{a}^{\prime}\right)$, which was previously shown to be equivalent to (a).

A.3. Unitary scalar products. Finally, we prove the equivalence of a second set of scalar product space properties. We adopt the name "unitary" for the scalar products satisfying these properties because of (b) and (e) in Theorem A.7.

DEFINITION A.6 (unitary scalar product). A scalar product is said to be unitary if it satisfies any one (and hence all) of the six equivalent properties in Theorem A.7.

THEOREM A.7. For a scalar product $\langle\cdot, \cdot\rangle_{\mathrm{M}}$ on $\mathbb{K}^{n}$, the following are equivalent:

(a) $\left(A^{*}\right)^{\star}=\left(A^{\star}\right)^{*}$ for all $A \in \mathbb{K}^{n \times n}$.

(a') $\left(A^{*}\right)^{\star}=\left(A^{\star}\right)^{*}$ for all $A$ in some CS-set for $\mathbb{K}^{n \times n}$.

(b) Adjoint preserves unitarity: $U$ unitary $\Rightarrow U^{\star}$ is unitary.

(c) Adjoint preserves Hermitian structure: H Hermitian $\Rightarrow H^{\star}$ is Hermitian. 
(d) Adjoint preserves Hermitian positive (semi)definite structure: H Hermitian positive (semi)definite $\Rightarrow H^{\star}$ is Hermitian positive (semi)definite.

(e) $M=\beta U$ for some unitary $U$ and $\beta>0$.

Proof. From (2.2) it follows that

$$
\left(A^{*}\right)^{\star}= \begin{cases}M^{-1} \bar{A} M & \text { for bilinear forms, } \\ M^{-1} A M & \text { for sesquilinear forms }\end{cases}
$$

and

$$
\left(A^{\star}\right)^{*}= \begin{cases}M^{*} \bar{A} M^{-*} & \text { for bilinear forms, } \\ M^{*} A M^{-*} & \text { for sesquilinear forms. }\end{cases}
$$

Thus for any individual matrix $A \in \mathbb{K}^{n \times n}$ we have

$$
\left(A^{*}\right)^{\star}=\left(A^{\star}\right)^{*} \Longleftrightarrow \begin{cases}\bar{A}\left(M M^{*}\right)=\left(M M^{*}\right) \bar{A} & \text { for bilinear forms, } \\ A\left(M M^{*}\right)=\left(M M^{*}\right) A & \text { for sesquilinear forms. }\end{cases}
$$

(a) $\Leftrightarrow\left(\mathrm{a}^{\prime}\right)$

(a) $\Rightarrow$ (a'): This implication is trivial.

(a') $\Rightarrow$ (a): Suppose $\left(A^{*}\right)^{\star}=\left(A^{\star}\right)^{*}$ holds for all $A$ in some CS-set for $\mathbb{K}^{n \times n}$. Then from (A.3) we conclude that $M M^{*}=\alpha I$ and hence that the two sides of (A.3) hold for all $A \in \mathbb{K}^{n \times n}$.

(a) $\Leftrightarrow(\mathrm{b})$

(a) $\Rightarrow$ (b): $U^{*}=U^{-1} \Rightarrow\left(U^{*}\right)^{\star}=\left(U^{-1}\right)^{\star} \stackrel{(\text { a })}{\Longrightarrow}\left(U^{\star}\right)^{*}=\left(U^{\star}\right)^{-1} \Rightarrow U^{\star}$ is unitary.

(b) $\Rightarrow$ (a): Suppose $U$, and hence also $U^{\star}$, is unitary. Then we have $\left(U^{\star}\right)^{*}=$ $\left(U^{\star}\right)^{-1}=\left(U^{-1}\right)^{\star}=\left(U^{*}\right)^{\star}$, showing that $\left(A^{*}\right)^{\star}=\left(A^{\star}\right)^{*}$ for all unitary $A$. But from Lemma A.2(f), the set of all unitaries is a CS-set for $\mathbb{K}^{n \times n}$; thus (a') holds, and hence also (a).

(a) $\Leftrightarrow(\mathrm{c})$

(a) $\Rightarrow(\mathrm{c}): H^{*}=H \Rightarrow\left(H^{*}\right)^{\star}=H^{\star} \stackrel{(\mathrm{a})}{\Longrightarrow}\left(H^{\star}\right)^{*}=H^{\star} \Rightarrow H^{\star}$ is Hermitian.

(c) $\Rightarrow$ (a): Suppose $H$, and therefore also $H^{\star}$, is Hermitian. Then we have $\left(H^{\star}\right)^{*}=H^{\star}=\left(H^{*}\right)^{\star}$, and thus $\left(A^{*}\right)^{\star}=\left(A^{\star}\right)^{*}$ for all Hermitian $A$. But from Lemma A.2(f), the set of all Hermitian matrices is a CS-set for $\mathbb{K}^{n \times n}$; thus (a') holds, and hence also (a).

$\underline{(\mathrm{a}) \Leftrightarrow(\mathrm{d})}$

(a) $\Rightarrow$ (d): Because (a) $\Rightarrow$ (c), we just need to show that positive (semi)definiteness is preserved by adjoint. But for $H$ Hermitian, $H^{\star}$ and $H$ are similar by definition of the adjoint and hence the eigenvalues of $H^{\star}$ and $H$ are the same.

$(d) \Rightarrow(a)$ : This argument is the same as that for (c) $\Rightarrow$ (a), using the fact that the set of all Hermitian positive (semi)definite matrices is a CS-set for $\mathbb{K}^{n \times n}$.

(a) $\Leftrightarrow(\mathrm{e})$

(a) $\Rightarrow$ (e): Suppose $\left(A^{*}\right)^{\star}=\left(A^{\star}\right)^{*}$ holds for all $A \in \mathbb{K}^{n \times n}$. Then we can conclude from (A.3) that $M M^{*}=\alpha I$ for some $\alpha \in \mathbb{K}$, and thus from Lemma A.3 that $M=\beta U$ for some unitary $U$ and $\beta>0$.

(e) $\Rightarrow$ (a): $M=\beta U \Rightarrow M M^{*}=(\beta U)\left(\bar{\beta} U^{*}\right)=\beta^{2} I$. Then by (A.3) we have $\left(A^{*}\right)^{\star}=\left(A^{\star}\right)^{*}$ for all $A$. 


\section{REFERENCES}

[1] G. Ammar, C. Mehl, and V. Mehrmann, Schur-like forms for matrix Lie groups, Lie algebras, and Jordan algebras, Linear Algebra Appl., 287 (1999), pp. 11-39.

[2] P. Benner, V. Mehrmann, And H. Xu, A numerically stable, structure preserving method for computing the eigenvalues of real Hamiltonian or symplectic pencils, Numer. Math., 78 (1998), pp. 329-358.

[3] R. Bhatia, Matrix factorizations and their perturbations, Linear Algebra Appl., 197/198 (1994), pp. 245-276.

[4] $\dot{A}$. Björck And S. Hammarling, A Schur method for the square root of a matrix, Linear Algebra Appl., 52/53 (1983), pp. 127-140.

[5] Y. Bolshakov and B. ReIChSTEIN, Unitary equivalence in an indefinite scalar product: An analogue of singular-value decomposition, Linear Algebra Appl., 222 (1995), pp. 155-226.

[6] Y. Bolshakov, C. V. M. van der Mee, A. C. M. Ran, B. Reichstein, and L. Rodman, Extension of isometries in finite-dimensional indefinite scalar product spaces and polar decompositions, SIAM J. Matrix Anal. Appl., 18 (1997), pp. 752-774.

[7] Y. Bolshakov, C. V. M. van der Mee, A. C. M. Ran, B. Reichstein, and L. Rodman, Polar decompositions in finite dimensional indefinite scalar product spaces: General theory, Linear Algebra Appl., 261 (1997), pp. 91-141.

[8] A. Bunse-Gerstner, R. Byers, and V. Mehrmann, A chart of numerical methods for structured eigenvalue problems, SIAM J. Matrix Anal. Appl., 13 (1992), pp. 419-453.

[9] A. Bunse-Gerstner And W. B. GragG, Singular value decompositions of complex symmetric matrices, J. Comput. Appl. Math., 21 (1988), pp. 41-54.

[10] A. ChaIYAKarn, Structure Preserving Algorithms for Computing the Symplectic Singular Value Decomposition, Ph.D. thesis, Western Michigan University, Kalamazoo, MI, 2005.

[11] L. Dieci, C. Elia, And L. Lopez, Smooth SVD on the Lorentz group with application to computation of Lyapunov exponents, J. Comput. Appl. Math., 164-165 (2004), pp. 255264.

[12] D. Ž. Djoković, J. Patera, P. Winternitz, and H. Zassenhaus, Normal forms of elements of classical real and complex Lie and Jordan algebras, J. Math. Phys., 24 (1983), pp. 13631374.

[13] D. Đoković, On the product of two alternating matrices, Amer. Math. Monthly, 98 (1991), pp. 935-936.

[14] H. Fassbender and K. Ikramov, Several observations on symplectic, Hamiltonian, and skewHamiltonian matrices, Linear Algebra Appl., 400 (2005), pp. 15-29.

[15] H. Fassbender, D. S. MaCkey, And N. MaCKey, Hamilton and Jacobi come full circle: Jacobi algorithms for structured Hamiltonian problems, Linear Algebra Appl., 332-334 (2001), pp. $37-80$.

[16] H. Fassbender, D. S. Mackey, N. Mackey, and H. XU, Hamiltonian square roots of skewHamiltonian matrices, Linear Algebra Appl., 287 (1999), pp. 125-159.

[17] H. Fassbender, D. S. Mackey, N. Mackey, and H. Xu, Real and Complex Hamiltonian Square Roots of Skew-Hamiltonian Matrices, Tech. report 92, Department of Mathematics \& Statistics, Western Michigan University, Kalamazoo, MI, 1999.

[18] I. Gohberg, P. Lancaster, and L. Rodman, Matrices and Indefinite Scalar Products, Oper. Theory Adv. Appl. 8, Birkhäuser-Verlag, Basel, Switzerland, 1983.

[19] G. H. Golub and C. F. Van Loan, Matrix Computations, 3rd ed., The Johns Hopkins University Press, Baltimore, MD, 1996.

[20] N. J. Higham, The matrix sign decomposition and its relation to the polar decomposition, Linear Algebra Appl., 212/213 (1994), pp. 3-20.

[21] N. J. Higham, Stable iterations for the matrix square root, Numer. Algorithms, 15 (1997), pp. $227-242$.

[22] N. J. Higham, J-orthogonal matrices: Properties and generation, SIAM Rev., 45 (2003), pp. 504-519.

[23] N. J. Higham, D. S. Mackey, N. Mackey, and F. Tisseur, Computing the polar decomposition and the matrix sign decomposition in matrix groups, SIAM J. Matrix Anal. Appl., 25 (2004), pp. 1178-1192.

[24] N. J. Higham, D. S. Mackey, N. Mackey, and F. Tisseur, Functions preserving matrix groups and iterations for the matrix square root, SIAM J. Matrix Anal. Appl., 26 (2005), pp. 849-877.

[25] R. A. Horn and C. R. Johnson, Matrix Analysis, Cambridge University Press, Cambridge, UK, 1985.

[26] R. A. Horn And C. R. Johnson, Topics in Matrix Analysis, Cambridge University Press, Cambridge, UK, 1991. 
[27] R. A. Horn And D. I. Merino, Contragredient equivalence: A canonical form and some applications, Linear Algebra Appl., 214 (1995), pp. 43-92.

[28] R. A. Horn, D. I. Merino, and D. Serre, The S-Orthogonal Groups, manuscript, 2004.

[29] K. D. Ikramov, Hamiltonian square roots of skew-Hamiltonian matrices revisited, Linear Algebra Appl., 325 (2001), pp. 101-107.

[30] A. ISERLES AND A. ZANNA, Efficient computation of the matrix exponential by generalized polar decompositions, SIAM J. Numer. Anal., 42 (2005), pp. 2218-2256.

[31] I. KAPlansky, Algebraic polar decomposition, SIAM J. Matrix Anal. Appl., 11 (1990), pp. 213217.

[32] C. Kenney and A. J. Laub, Rational iterative methods for the matrix sign function, SIAM J. Matrix Anal. Appl., 12 (1991), pp. 273-291.

[33] U. Kintzel, Procrustes problems in finite dimensional indefinite scalar product spaces, Linear Algebra Appl., 402 (2005), pp. 1-28.

[34] S. Lang, Linear Algebra, 3rd ed., Springer-Verlag, New York, 1987.

[35] J. D. Lawson, Polar and Ol'shanskii decompositions, J. Reine Angew. Math., 448 (1994), pp. 191-219.

[36] W.-W. Lin, V. Mehrmann, and H. Xu, Canonical forms for Hamiltonian and symplectic matrices and pencils, Linear Algebra Appl., 302/303 (1999), pp. 469-533.

[37] D. S. Mackey and N. Mackey, On the Determinant of Symplectic Matrices, Numerical Analysis Report 422, Manchester Centre for Computational Mathematics, Manchester, UK, 2003.

[38] D. S. Mackey, N. Mackey, and D. M. Dunlavy, Structure preserving algorithms for perplectic eigenproblems, Electron. J. Linear Algebra, 13 (2005), pp. 10-39.

[39] D. S. Mackey, N. Mackey, and V. Mehrmann, Analytic SVD of Symplectic Matrices, Tech. report, Technische Universität Berlin, Berlin, Germany, 2004, in preparation.

[40] C. MeHL, Condensed forms for skew-Hamiltonian/Hamiltonian pencils, SIAM J. Matrix Anal. Appl., 21 (1999), pp. 454-476.

[41] C. Mehl, V. Mehrmann, and H. Xu, Canonical forms for doubly structured matrices and pencils, Electron. J. Linear Algebra, 7 (2000), pp. 112-151.

[42] C. Mehl, A. C. M. Ran, and L. Rodman, Polar decompositions of normal operators in indefinite inner product spaces, in Operator Theory in Krein Spaces and Nonlinear Eigenvalue Problems, Oper. Theory Adv. Appl. 162, Birkhäuser-Verlag, Basel, Switzerland, 2006, pp. 277-292.

[43] V. Mehrmann And H. Xu, Structured Jordan canonical forms for structured matrices that are Hermitian, skew-Hermitian, or unitary with respect to indefinite inner products, Electron. J. Linear Algebra, 5 (1999), pp. 67-103.

[44] V. L. Mehrmann, The Autonomous Linear Quadratic Control Problem-Theory and Numerical Solution, Lecture Notes in Control and Inform. Sci. 163, Springer-Verlag, Berlin, 1991.

[45] K. R. Meyer and G. R. HAll, Introduction to Hamiltonian Dynamical Systems and the NBody Problem, Appl. Math. Sci. 90, Springer-Verlag, New York, 1991.

[46] H. Z. Munthe-KaAs, G. R. W. Quispel, and A. ZannA, Generalized polar decomposition on Lie groups with involutive automorphisms, Found. Comput. Math., 1 (2001), pp. 297-324.

[47] J. D. RoberTs, Linear model reduction and solution of the algebraic Riccati equation by use of the sign function, Internat. J. Control, 32 (1980), pp. 677-687.

[48] R. SHAw, Linear Algebra and Group Representations. Vol. I, Academic Press Inc. [Harcourt Brace Jovanovich], London, New York, 1982.

[49] H. Xu, An SVD-like matrix decomposition and its applications, Linear Algebra Appl., 368 (2003), pp. 1-24.

[50] T. Yamanouchi, Quantum mechanics, in Mathematics Applied to Physics, E. Roubine, ed., Springer-Verlag, New York, 1970, pp. 562-601.

[51] A. Zanna And H. Z. Munthe-KaAs, Generalized polar decompositions for the approximation of the matrix exponential, SIAM J. Matrix Anal. Appl., 23 (2002), pp. 840-862. 